\title{
PRICE DECISIONS WITH ASYMMETRIC REFERENCE EFFECT AND INFORMATION SHARING IN DUAL-CHANNEL SUPPLY CHAINS
}

\author{
NengGui ZhaO ${ }^{1,2}$, Qiang Wang,* AND JiE Wu ${ }^{3}$
}

\begin{abstract}
This paper considers a retailer-manufacturer dual-channel supply chain (DCSC) consisting of a retail channel and a direct sale channel. Previous literature has shown that either asymmetric reference effect (ARE) or information sharing (IS) significantly impacts customers' demand, then the channel members' pricing decisions. As yet, no literature has examined the joint effect of both on the channel members' pricing decisions, especially in a DCSC. To fill up the deficiency, we first explore and compare the pricing decisions in a centralized and a decentralized DCSC with ARE, respectively, with and without IS, using the Stackelberg game and two-stage optimization technique. Then we evaluate the values of ARE and IS by introducing model misspecification and numerical experiments. We find that substantial revenue will be lost if the retailer ignores ARE when information is shared than not shared, especially when the channel members are pessimistic about the market. A higher reference price or a weaker ARE induces the channel members to increase prices, and make IS more valuable to them. Besides, whether the information is shared or not, channel members generally underestimate revenues under a relatively high reference price, while overestimating the revenues under a relatively small reference price. Furthermore, the manufacturer conditionally accepts the IS while the retailer always accepts it.
\end{abstract}

Mathematics Subject Classification. 91A40, 91B50.

Received May 18, 2021. Accepted February 5, 2022.

\section{INTRODUCTION}

As the Internet information technology develops rapidly, modern customers are increasingly accustomed to shopping online, which induces many manufacturers to add a direct sale channel as a strategy to attract customers and improve profits. For example, in many clothing enterprises, like Zara and Uniqlo, customers could purchase their products from entity shops or online. Chiang et al. [6] reported that about $42 \%$ of manufacturers sell their products directly online to customers. It is not only manufacturers that are increasingly selling products through two different channels (traditional retail and direct sale), but also retailers are gradually conscious of the advantages of the dual-channel sales model [11].

Keywords. Supply chain management, asymmetric reference effect, information sharing, dual-channel, Stackelberg game.

1 School of Management, Hefei University of Technology, Hefei 230009, P.R. China.

2 Key Laboratory of Process Optimization and Intelligent Decision-Making of Ministry of Education, Hefei 230009, P.R. China.

3 International Institute of Finance, School of Management, University of Science and Technology of China, Hefei 230026,

P.R. China.

${ }^{*}$ Corresponding author: qiangwan@mail.ustc.edu.cn

(C) The authors. Published by EDP Sciences, ROADEF, SMAI 2022 
Reference effect, an important marketing phenomenon that significantly influences customers purchasing behavior, has gained increasing attention over the last couple of decades. (e.g., [10,33]). Reference effect generally refers to reference price effect or reference quality effect according to the previous literature, and it refers to reference price effect in this paper. Reference price is generally regarded as an internal anchor and formed in customers' minds through the historical prices or the competitor's prices. Kalwani et al. [16] stated that reference prices are evolved in the customers' minds before they making purchasing decisions. When the current sale price is smaller than the reference price, they will feel a gain, which will clearly stimulate demand; Conversely, they will feel a loss, which will reduce the demand. With the rapid development of the economy and network, customers are becoming more and more "shrewd", and they will inevitably refer to the historical prices before making purchasing decisions, that is reference price exists in the customers' mind factually. Previous literature (e.g., [35]) stated that the reference price greatly influences the decision-makers' pricing decisions.

On the other hand, information sharing (IS), as a hot topic in supply chain management, has attracted wide attention from business comments in practice, it could help the decision-makers understand the market clearly, and further help them improve the accuracy of demand forecasting and then make more precise operational decisions. The existing literature has stated that IS could bring significant revenue for the retailer, for example, Raju and Roy [24] stated that accurate market information could help the decision-makers significantly improve the profits. Hosoda et al. [12] investigated the advantages of sharing the market information by real-life data sets, and they stated that sharing information can cut inventory costs by $8 \%-19 \%$. Whereas, there exists literature stating that IS is not always supported by channel members, for example, Zhang et al. [35] explored a problem of demand forecast sharing in a supply chain, both the retailer and the manufacturer could conduct demandenhancing service. The authors stated that when the former conducts service, no IS is the best; When the latter conducts service, if the service efficiency is high (low), the former would share (not share) demand information. From the above mentioned literature, we obtain that whether IS enables the channel members to achieve a win-win depends on the research background.

Subsequently, an interesting research topic emerges, whether IS enables the channel members in a DCSC with ARE to achieve win-win? Or, does ARE has a consistent impact on the system, regardless of IS? Based on these issues, in this paper we jointly consider ARE and IS in a DCSC, the topic is motivated by the fact that both of these two factors significantly influence customers' demand, then the channel members' pricing decisions and revenues. Besides, both the theoretical and numerical results show that just considering one factor in this paper will result in the channel members making inaccurate decisions and suffering substantial losses. As yet, no literature has explored the joint effect of them on the pricing decisions, and this work tries to bridge the gap. It is widely cognized that two key elements impact consumers' purchasing behaviors profoundly in a supply chain, one is the consumers' individual behaviors, e.g., reference behaviors, social learning, strategic behaviors, etc; the other is the retailers ${ }^{11}$ policies, e.g., demand learning, price guarantee, revenue-sharing contract, etc. Simultaneously considering these two elements are more in line with the actual situation, and it has been widely studied in the existing literature (e.g., $[2,37])$.

In this paper, we first separately derive the optimal pricing decisions in a centralized and a decentralized DCSC with and without IS. Then we examine the values of ARE and IS to the centralized and decentralized scenarios, respectively. In the centralized scenario, the channel members have a common goal, i.e., maximizing the revenues of the entire scenario; whereas, in the decentralized scenario, the channel members are committed to maximizing their individual revenues. We formulate the problem in the decentralized scenario as a Stackelberg game and solve it utilizing the game theory and optimization method. In such a game, the manufacturer makes pricing decisions firstly, then the retailer makes pricing decisions according to the manufacturer's decisions.

The contributions of this work are mainly embodied in the following two points: First, our work is the first to jointly consider ARE and IS in a DCSC that consists of a retail and a direct sale channel, the topic is motivated by the fact that both of them significantly influence customers' demand, especially the demand that comes from the retail channel, then influence the channel members' pricing decision. We believe this paper will be a

\footnotetext{
${ }^{1}$ It is worth noting that here "retailers" refers to the supply chain members except consumers, for example, manufacturers, retailers, sellers, and distributors, etc.
} 
complement to the literature on DCSCs. Second, we introduce a new method, i.e., model misspecification, to further investigate the values of ARE to the centralized and decentralized DCSCs with and without IS. The method is based on the fact that ARE exists in the consumers' minds, while the channel members generally ignore it, which in turn affects their pricing behaviors significantly.

The rest of our work is arranged as follows. In Section 2, we review the related literature to our study. In Section 3, we present model description and notation. In Section 4, we respectively derive the optimal pricing decisions in a centralized and a decentralized DCSC with ARE. In Section 5, we extend the model of Section 4 by considering IS. In Section 6, we investigate the values of ARE to the supply chain. In Section 7, we evaluate the performance of sharing information. In Section 8, we conclude the paper.

\section{Related Literature}

This paper focuses on exploring the optimal pricing decisions with asymmetric reference effect (ARE) and information sharing (IS) in a dual-channel supply chain. The most representative and relevant literature about (asymmetric) reference price, information sharing, and dual-channel supply chain (DCSC) to our study are reviewed. We summarize three main streams

The first stream focuses on pricing decisions in a DCSC. In the past few decades, pricing decisions in DCSCs have gained ascending attention from business and supply chain researchers. Huang and Swaminathan [14] presented a pricing model in a supply chain with a retail channel and an online sale channel, the authors assumed the demand is deterministic. Hua et al. [13] simultaneously explored the optimal pricing and delivery lead time decisions in a DCSC, the authors took lead time into account because they believe it significantly affects customers' demands. One distinct feature of our paper is that we consider ARE into a DCSC system because we believe it also significantly affects customers' demands. Li et al. [20] explored the optimal pricing decisions in a DCSC with a risk-averse retailer and a risk-neutral manufacturer, in which demand is stochastic and price-dependent. Chen et al. [4] proposed a pricing and quality decision-making problem, in which the authors assumed the product can be sold through three ways: traditional retail channel, online channel, and dual-channel. Different from them, we assume the products can be delivered through a dual-channel consisting of a retail channel and an online channel. Chen et al. [5] studied the joint effect of retail service, manufacturer's direct service and quality effect on channel members' decisions in a DCSC that consists of a traditional retail channel and an online direct channel. Although pricing decisions in DCSCs have been extensively studied, there are still some important market phenomena that have not been involved, e.g., (asymmetric) reference effect.

Substantial literature focus on pricing decisions with (asymmetric) reference effect (see [17,22]). Fibich et al. [9] introduced a new two-stage method to derive the explicit pricing decision under ARE, they pointed out that ARE generally leads to a non-smooth problem and standard methods cannot be applied. Güler et al. [10], Xue et al. [31] and Dye and Yang [8] studied dynamic pricing problems, in which demand functions are dependent on ARE. Zhang et al. [33] presented a cooperative advertising model, where advertising affects the reference price, the authors investigated the impacts of $\mathrm{RE}$ on the pricing strategies of channel members. Zhang et al. [34] studied two advertising models to explore optimal pricing strategies for a firm, in two models advertising effect affects reference price through goodwill and the authors assume the reference price has a vital effect on consumer behaviors. Duan and Cao [7] studied a joint dynamic pricing and inventory management problem in which demand rate relies heavily on the selling price and customers' reference price and displayed stock quantity. Zhao et al. [36] explored a two-period pricing model with RE and strategic consumers, the authors assumed the reference price only affects the customers' behaviors in the second period. Cao et al. [2] proposed a dynamic pricing model with RE, in which demand information is not available to the retailer and will be studied by a Bayesian approach. However, for all we know, as yet there is no literature studied the influences of the asymmetric RE on the optimal pricing strategies in a DCSC. We believe this paper will be a complement to the literature on pricing decisions in DCSCs.

The third stream focuses on IS in a supply chain. For example, Rached et al. [23] studied the influences of sharing different types of information on a serial supply chain that consists of a supplier, warehouse, retailer, 
and customers. Huang et al. [15] studied a retailer's incentive for sharing information with a manufacturer who may encroach the market, and they showed that information sharing can be an effective way for deterring manufacturer encroachment. As far as we know, most of the research on IS concentrates on sharing demand data. For example, Cachon and Fisher [1] and Wang et al. [29] considered the values of sharing information on inventory and demand in a supply chain, by comparing a traditional supply chain without IS. Lee et al. [18] suggested that IS is of great value to a two-level supply chain, especially when the demands are significantly correlated over time. Leng and Parlar [19] analyzed an allocated cost-saving problem, where information on demand is shared in a three-level supply chain that consists of one retailer, one manufacturer, and one distributor. Zhu [38] outsourcing presented an outsourcing model, in which an asymmetric demand information issue exists between the supplier and the buyer. The authors obtained the conditions for sharing demand information.

One research purpose of this paper is to examine the performance of IS, which directly influences the demand function. Market information generally can be identified as one specific case of demand information. Previous literature (e.g., [24]) shows that a piece of more accurate market information has a greater impact on profits, especially in a Stackelberg model. Hosoda et al. [12] investigated the benefits of sharing market sales information using a real-life data set, they revealed that sharing market information can cut inventory costs by between $8 \%$ and $19 \%$. Ma et al. [21] revealed that the buyers in the market can improve their demand forecast by obtaining more market information.

The literature mentioned above suggests that both IS and (asymmetric) reference effect individually impact the channel members' demand significantly, and both of them have been studied deeply over the past several decades. As yet, there is no literature that jointly considers both of them, especially in a DCSC. This paper extends the existing literature by combining ARE and IS, discussing the joint impact on the optimal pricing decisions, and providing interesting managerial insights for channel members.

\section{MOdEL DESCRIPTION}

Consider a retailer-manufacturer dual-channel supply chain (DCSC) that consists of a traditional retail channel and a direct sale (online) channel. The manufacturer can first sell products to the retailer at a wholesale price, i.e., $w$, then the retailer directly sells products to the customers at retail price, i.e., $p_{r}$; or directly sell to customers at a direct sale price, i.e., $p_{d}$ (see Fig. 1). The retailer is generally closer to the consumers and has more expertise and superior forecasting abilities than the manufacturer in the selling process, thus he ${ }^{2}$ holds a better knowledge about the information on market size and customers' behaviors than the manufacturer. In this paper, we assume that the retailer knows the full information about the market size; while the manufacturer just knows the partial information about the market, i.e., the distribution of the market size.

One research purpose of this paper is to explore the value of sharing the market information between the two players (i.e., the retailer and the manufacturer) to a DCSC, and thus we analyze the problem in the following two cases: (i) Non-information sharing (NI): the retailer knows the full information about the market size, whereas the manufacturer just knows the partial information about the market size; (ii) Information sharing (IS): the retailer is willing to share the market information with the manufacturer, but a contract or a commitment exists between them, i.e., once the manufacturer accepts the information, she must pay a certain fee to the retailer. In either case, we start our analysis in a centralized DCSC, where the channel members have a common goal, i.e., maximizing the revenue of the entire DCSC by optimizing their own pricing policies $\left(p_{r}, p_{d}\right.$, and $\left.w\right)$; Then we analyze the problem in a decentralized DCSC, where the channel members are committed to maximizing their individual revenues. We characterize the problem in the decentralized scenario to be a Stackelberg game, and then use game theory and two-stage optimization methods to solve it. In this game, the manufacturer first decides the direct sale price and the wholesale price, then the retailer decides the retail price in response to the manufacturer's pricing decisions. Besides, we assume the direct sale price should be larger than the wholesale price, i.e., $p_{d} \geq w$, otherwise the retailer would order products via the direct sale channel by a cheaper price;

\footnotetext{
${ }^{2}$ For convenience, we use "she" denotes the manufacturer and "he" denotes the retailer in the following.
} 


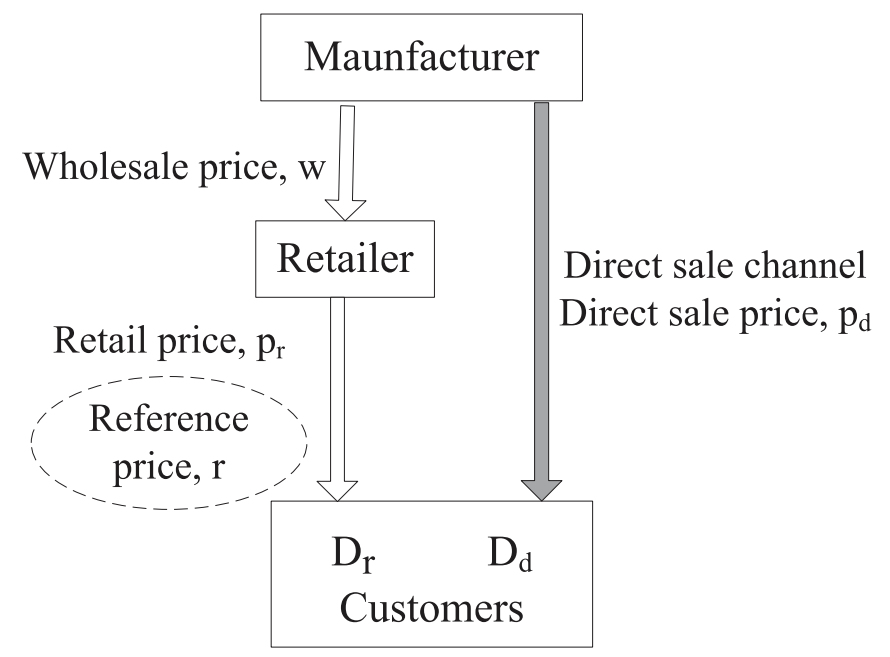

FIGURE 1. Diagrammatic sketch of a dual-channel system.

Note that the retail price should be larger than the wholesale price, i.e., $p_{r} \geq w$, otherwise it is unprofitable for the retailer.

\subsection{Basic model}

We assume consumers' demand comes from the retail (resp. direct sale) channel, i.e., $d_{r}$ (resp. $d_{d}$ ), is positive linear in the retail (resp. direct sale) price but negative linear in the direct sale (resp. retail) price (see [24]). Thus, the base demand function can be formulated as

$$
d_{r}=\theta a-b_{1} p_{r}+\beta_{1} p_{d} \text { and } d_{d}=(1-\theta) a-b_{2} p_{d}+\beta_{2} p_{r}
$$

where $a \in\left\{a_{\mathrm{L}}, a_{\mathrm{H}}\right\}\left(a_{\mathrm{L}}<a_{\mathrm{H}}\right)$ is the market size, it equals to $a_{\mathrm{L}}$ with probability $\lambda \in(0,1)$ and equals to $a_{\mathrm{H}}$ with probability $1-\lambda ; \theta$ measures the customers' preference to the retail channel, a larger $\theta$ implies that more customers choose to purchase the products through the retail channel; $b_{1}$ and $b_{2}$ are the self-price coefficients of $d_{r}$ and $d_{d}$, respectively, and $\beta_{i}$ reflects the cross-price coefficients. To facilitate the analysis of the model, we define $\beta_{1}=\beta_{2}:=\beta$. Furthermore, we assume $b_{i} \geq \beta$ in light of the self-price coefficient is larger than cross-price effect.

Consumers in the market are assumed strategic, they generally form a reference price $r$ in their minds after observing the historical prices of the product, the formed reference price $r$ will inevitably affect their purchasing behaviors at the current selling price. We assume reference price has a direct effect, i.e., reference effect, on the customers' demand, which has been widely applied in the previous literature (e.g., $[3,34])$. In this paper we consider reference price is evolved from the retail prices and it affects the demand comes from the retail channel ${ }^{3}$ Thus, we rewrite the demands under the asymmetric reference effect(ARE) from the two channels (i.e., $D_{r}$ and $\left.D_{d}\right)$ as follows:

$$
D_{r}=\theta a-b_{1} p_{r}+\beta p_{d}+\eta\left(r-p_{r}\right) \text { and } D_{d}=(1-\theta) a-b_{2} p_{d}+\beta p_{r},
$$

\footnotetext{
${ }^{3}$ We extend the original model by considering that the reference price also appears in the direct channel in Appendix B, the extended model shows that considering the reference price in the direct channel has little influence on the exploration of the reference effect, e.g., the structures of the optimal prices and revenues are similar to that in the original model, except changing some parameters. However, the optimal decisions will be extremely complicated if we extend the model, thus the loss outweighs the gain. Besides, the research perspective on RE is similar to Wang et al. [30] and Sun et al. [27] if we set the direct sales price $p_{d}$ as the reference price $r$. In other words, their setting of reference price can be regarded as a special case of ours.
} 
where $\eta \in\left\{\eta^{+}, \eta^{-}\right\} \geq 0$, represents the intensity of ARE. Specifically, $\eta=\eta^{+}$if $r \geq p_{r}$, otherwise $\eta=\eta^{-}$. Notice that $\eta=0$ denotes that there is no ARE in the supply chain. Generally, the types of customers are classified into loss-averse, gain-seeking or loss-neutral, which depend on $\eta^{+}<\eta^{-}, \eta^{+}>\eta^{-}$, or $\eta^{+}=\eta^{-}$. Prospect theory [28] postulates that loss aversion behavior is more common than loss-seeking behavior, and thus we focus on the loss-averse case in this paper, i.e., $\eta^{+}<\eta^{-}$. Furthermore, it is reasonable to define the demands for the two channels are nonnegative, i.e., $D_{r} \geq 0$ and $D_{d} \geq 0$, then we obtain

$$
p_{r} \leq \frac{\theta a b_{2}+(1-\theta) a \beta+b_{2} \eta r}{\left(b_{1}+\eta\right) b_{2}-\beta^{2}} \text { and } p_{d} \leq \frac{\theta a \beta+(1-\theta) a\left(b_{1}+\eta\right)+\beta \eta r}{\left(b_{1}+\eta\right) b_{2}-\beta^{2}} .
$$

It is rational to suppose that the largest reference price is not larger than the largest feasible price, combining that the reference price is evolved from the retail prices in this paper, thus we have $r_{\max } \leq \frac{\theta a b_{2}+(1-\theta) a \beta+b_{2} \eta r_{\max }}{\left(b_{1}+\eta\right) b_{2}-\beta^{2}}$, i.e., $r_{\max } \leq \frac{\theta a b_{2}+(1-\theta) a \beta}{b_{1} b_{2}-\beta^{2}}$.

Define $\Pi_{m}$ and $\Pi_{r}$ as the revenues ${ }^{4}$ of the manufacturer and the retailer, respectively, $\Pi$ as revenue of the entire DCSC. Then, $\Pi_{m}, \Pi_{r}$ and $\Pi$ can be determined as

$$
\begin{aligned}
\Pi_{m} & =w D_{r}+p_{d} D_{d}, \\
\Pi_{r} & =\left(p_{r}-w\right) D_{r}, \\
\Pi & =\Pi_{m}+\Pi_{r}=p_{r} D_{r}+p_{d} D_{d} .
\end{aligned}
$$

\subsection{Model misspecification}

Note that one interesting and important purpose of this work is to explore the influences of ARE on the channel members' pricing decisions and revenues. Here, we introduce model misspecification to clearly explore the influence. Model misspecification was initially proposed to test model errors, for example, Signorino and Yilmaz [26] proposed the model errors in respect to parameters and data, whereas the error in this work refers to the background (see [35] for a similar reference). Specifically, as the economy and Internet rapidly develop, customers become more and more "shrewd" in their purchase process, and they are accustomed to consulting the products' historical prices, that is RE exists in the customers' mind factually. However, the retailer may ignore (or do not realize) the fact, so what happens to the channel members' pricing decisions if RE is ignored?

Denote $\bar{w}, \bar{p}_{d}$ and $\bar{p}_{r}$ as the corresponding wholesale, direct sale, and retail prices if RE is ignored (i.e., $\left.\eta^{+}, \eta^{-}=0\right)$. The demands from the two channels that the channel members believe are

$$
\bar{D}_{r}=\theta a-b_{1} \bar{p}_{r}+\beta \bar{p}_{d} \text { and } \bar{D}_{d}=(1-\theta) a-b_{2} \bar{p}_{d}+\beta \bar{p}_{r} .
$$

Since RE exists factually, the actual demands that they would obtain are

$$
\tilde{D}_{r}=\theta a-b_{1} \bar{p}_{r}+\beta \bar{p}_{d}+\eta\left(r-\bar{p}_{r}\right) \text { and } \tilde{D}_{d}=\bar{D}_{d} .
$$

Denote $\bar{\Pi}_{m}$ (resp. $\bar{\Pi}_{r}$ ) and $\tilde{\Pi}_{m}$ (resp. $\tilde{\Pi}_{r}$ ) as the revenues the manufacturer(resp. retailer) expects to obtain and she (resp. he) actually would obtain, respectively, then

$$
\begin{aligned}
\bar{\Pi}_{m} & =\bar{w} \bar{D}_{r}+\bar{p}_{d} \bar{D}_{d}, \tilde{\Pi}_{m}=\bar{w} \tilde{D}_{r}+\bar{p}_{d} \tilde{D}_{d}, \\
\bar{\Pi}_{r} & =\left(\bar{p}_{r}-\bar{w}\right) \bar{D}_{r}, \tilde{\Pi}_{r}=\left(\bar{p}_{r}-\bar{w}\right) \tilde{D}_{r} .
\end{aligned}
$$

The revenues of the entire centralized DCSC that the channel members expect to obtain $\bar{\Pi}$, and they actually would obtain $\tilde{\Pi}$, can be formulated as

$$
\bar{\Pi}=\bar{p}_{r} \bar{D}_{r}+\bar{p}_{d} \bar{D}_{d} \text { and } \tilde{\Pi}=\bar{p}_{r} \tilde{D}_{r}+\bar{p}_{d} \tilde{D}_{d}
$$

We summarize notation in Table 1 for later convenience.

\footnotetext{
${ }^{4}$ It follows from Shen et al. [25] that we assume the unit ordering cost is 0, because a pricing model with an ordering cost can be transformed equivalently into another pricing model in which the ordering cost is 0 but the other cost parameters need to be modified correspondingly.
} 
TABLE 1. Notation.

\begin{tabular}{ll}
\hline \hline Notation & Description \\
\hline$a \in\left\{a_{\mathrm{L}}, a_{\mathrm{H}}\right\}$ & Market size \\
$b_{i}, i=1,2$ & Self-efficiency of price \\
$\beta$ & Cross-efficiency of price \\
$\eta \in\left\{\eta^{+}, \eta^{-}\right\}$ & Intensity of ARE \\
$r$ & Reference price \\
$k$ & Unit information cost \\
$\lambda$ & Probability that $a=a_{\mathrm{L}}$ \\
$p_{r}\left(\right.$ resp. $\left.\bar{p}_{r}\right)$ & Retail price with(resp. without) ARE \\
$p_{d}\left(\right.$ resp. $\left.\bar{p}_{d}\right)$ & Direct sale price with(resp. without) ARE \\
$w($ resp. $\bar{w})$ & Wholesale price with(resp. without) ARE \\
$D_{r}\left(\right.$ resp. $\left.\bar{D}_{r}\right)$ & Demand comes from retail channel with(resp. without) ARE \\
$\tilde{D}_{r}$ & Actual demand comes from retail channel if RE is ignored \\
$D_{d}\left(\right.$ resp. $\left.\bar{D}_{d}\right)$ & Demand comes from direct sale channel with(resp. without) ARE \\
$\tilde{D}_{d}$ & Actual demand comes from direct sale channel if ARE is ignored \\
$\bar{\Pi}_{m} / \tilde{\Pi}_{m} / \hat{\Pi}_{m}$ & Manufacturer's expected/actual/optimal revenue \\
$\bar{\Pi}_{r} / \tilde{\Pi}_{r} / \hat{\Pi}_{r}$ & Retailer's expected/actual/optimal revenue \\
$\bar{\Pi}_{c} / \tilde{\Pi}_{c} / \hat{\Pi}_{c}$ & Total expected/actual/optimal revenue of the centralized DCSC \\
\hline
\end{tabular}

Notes. Note that the above notation added a subscript "c" (resp. "d") denotes the corresponding notation in the centralized (resp. decentralized) DCSC, and added a superscript NI (resp. IS) denotes the corresponding notation in NI (resp. IS) case.

\section{Non-INFOrmation Sharing (NI) CASE}

In this section, we assume there is no information sharing between the channel members, the retailer knows the market size accurately, while the manufacturer only knows the distribution of the market size. Consider that the market size $a$ is ex ante random, which can be either high $\left(a=a_{\mathrm{H}}\right)$ with probability $\lambda$ or low $\left(a=a_{\mathrm{L}}\right)$ with probability $1-\lambda$, where $a_{\mathrm{H}}>a_{\mathrm{L}}>0$. Then the expected market size $\tilde{a}$ is given as $\tilde{a}=\lambda a_{\mathrm{L}}+(1-\lambda) a_{\mathrm{H}}$. Apparently, a higher $\lambda$ implies that the manufacturer is more negative about the market.

To avoid confusing notation, we add the superscript "NI" to the notation shown in Section 3 to denote the corresponding case in this section. Specifically, we define $w^{\mathrm{NI}}, p_{r}^{\mathrm{NI}}$ and $p_{d}^{\mathrm{NI}}$ as the corresponding pricing decisions, $D_{r}^{\mathrm{NI}}$ and $D_{d}^{\mathrm{NI}}$ as the corresponding demands, then

$$
D_{r}^{\mathrm{NI}}=\theta a-b_{1} p_{r}^{\mathrm{NI}}+\beta p_{d}^{\mathrm{NI}}+\eta\left(r-p_{r}^{\mathrm{NI}}\right) \text { and } D_{d}^{\mathrm{NI}}=(1-\theta) \tilde{a}-b_{2} p_{d}^{\mathrm{NI}}+\beta p_{r}^{\mathrm{NI}} .
$$

Define $\mathbb{E} \Pi_{m}^{\mathrm{NI}}$ and $\mathbb{E} \Pi_{r}^{\mathrm{NI}}$ as the expected revenues of the manufacturer and the retailer, respectively, $\mathbb{E} \Pi_{c}^{\mathrm{NI}}$ as the total expected revenue of DCSC, then

$$
\begin{aligned}
& \mathbb{E} \Pi_{m}^{\mathrm{NI}}=w^{\mathrm{NI}} D_{r}^{\mathrm{NI}}+p_{d}^{\mathrm{NI}} D_{d}^{\mathrm{NI}}, \quad \mathbb{E} \Pi_{r}^{\mathrm{NI}}=\Pi_{r}^{\mathrm{NI}}=\left(p_{r}^{\mathrm{NI}}-w^{\mathrm{NI}}\right) D_{r}^{\mathrm{NI}}, \\
& \mathbb{E} \Pi_{c}^{\mathrm{NI}}=\mathbb{E} \Pi_{m}^{\mathrm{NI}}+\mathbb{E} \Pi_{r}^{\mathrm{NI}}=p_{r}^{\mathrm{NI}} D_{r}^{\mathrm{NI}}+p_{d}^{\mathrm{NI}} D_{d}^{\mathrm{NI}} .
\end{aligned}
$$

\subsection{Centralized DCSC}

We first derive the optimal pricing decisions in a centralized DCSC, where the channel members aim to maximize the revenue of the entire DCSC, we then derive the optimal decisions if RE is ignored. It is immediately obtained from equation (4.2) that the total revenue is completely independent of the wholesale price, the channel members need to optimize the retail and the direct sale prices. To avoid confusion, we add a subscript "c" to the notation shown in Section 3 to denote the corresponding notation in the centralized DCSC, a subscript "d" is added to denote the corresponding notation in the decentralized DCSC. Specifically, we define $p_{r, c}^{\mathrm{NI}}$ and $p_{d, c}^{\mathrm{NI}}$ as the corresponding retail price and the direct sale price, respectively, $\Pi_{c}^{\mathrm{NI}}$ as the revenue of the entire DCSC. 
Proposition 4.1. In the centralized DCSC of NI case, $\mathbb{E} \Pi_{c}^{\mathrm{NI}}$ is jointly concave in $p_{r, c}^{\mathrm{NI}}$ and $p_{d, c}^{\mathrm{NI}}$; There exists two thresholds on reference price $\bar{r}_{c}^{\mathrm{NI}}=\frac{\theta a b_{2}+(1-\theta) \tilde{a} \beta}{2 b_{1} b_{2}+\eta^{+} b_{2}-2 \beta^{2}} \geq \underline{r}_{c}^{\mathrm{NI}}=\frac{\theta a b_{2}+(1-\theta) \tilde{a} \beta}{2 b_{1} b_{2}+\eta^{-} b_{2}-2 \beta^{2}}$, such that

(i) if $r \geq \bar{r}_{c}^{\mathrm{NI}}$, the optimal retail and direct sale prices are given by

$$
\hat{p}_{r, c}^{\mathrm{NI}}=\frac{1}{2} \frac{\theta a b_{2}+b_{2} \eta^{+} r+(1-\theta) \tilde{a} \beta}{\left(b_{1}+\eta^{+}\right) b_{2}-\beta^{2}} \text { and } \hat{p}_{d, c}^{\mathrm{NI}}=\frac{1}{2} \frac{\beta \theta a+\beta \eta^{+} r+(1-\theta) \tilde{a}\left(b_{1}+\eta^{+}\right)}{\left(b_{1}+\eta^{+}\right) b_{2}-\beta^{2}} ;
$$

(ii) if $r \leq \underline{r}_{c}^{\mathrm{NI}}$, the optimal retail and direct sale prices are given by

$$
\hat{p}_{r, c}^{\mathrm{NI}}=\frac{1}{2} \frac{\theta a b_{2}+b_{2} \eta^{-} r+(1-\theta) \tilde{a} \beta}{\left(b_{1}+\eta^{-}\right) b_{2}-\beta^{2}} \text { and } \hat{p}_{d, c}^{\mathrm{NI}}=\frac{1}{2} \frac{\beta \theta a+\beta \eta^{-} r+(1-\theta) \tilde{a}\left(b_{1}+\eta^{-}\right)}{\left(b_{1}+\eta^{-}\right) b_{2}-\beta^{2}} ;
$$

(iii) otherwise, the optimal retail and direct sale prices are given by

$$
\hat{p}_{r, c}^{\mathrm{NI}}=r \text { and } \hat{p}_{d, c}^{\mathrm{NI}}=\frac{(1-\theta) \tilde{a}+2 \beta r}{2 b_{2}} .
$$

Besides, the optimal expected revenue $\mathbb{E}_{c}^{\mathrm{NI}}$ is given by equation $(4.2)$ when $p_{r}^{\mathrm{NI}}=\hat{p}_{r, c}^{\mathrm{NI}}$ and $p_{d}^{\mathrm{NI}}=\hat{p}_{d, c}^{\mathrm{NI}}$.

The proofs of all theoretical results are presented in Appendix A. The joint concavity of the total expected revenue implies the uniqueness of the optimal prices. Proposition 4.1 indicates the optimal prices are piecewise functions due to the asymmetric RE. If RE is symmetric, i.e., $\bar{r}_{c}^{\mathrm{NI}}=\underline{r}_{c}^{\mathrm{NI}}$, then the optimal prices are continuous, which further implies the influence of the asymmetric RE on the pricing decisions is profound.

Proposition 4.2. The optimal retail price and direct sale price, $\hat{p}_{r, c}^{\mathrm{NI}}$ and $\hat{p}_{d, c}^{\mathrm{NI}}$, are (i) nonincreasing in $\eta^{+}$and $\eta^{-}$; (ii) increasing in $r$, and the increment rate of $\hat{p}_{r, c}^{\mathrm{NI}}$ is higher than that of $\hat{p}_{d, c}^{\mathrm{NI}}$; (iii) decreasing in $\lambda$.

Proposition 4.2 states some structural properties about the optimal prices. Specifically, the higher the reference price, or the smaller the intensity of $\mathrm{RE}$, the higher the retail and direct sale prices, which can be interpreted as follows: A higher reference price implies that customers are willing to pay more for the products, which naturally stimulates the channel members to charge higher prices to increase the revenue. The smaller the intensity of RE implies the smaller demand (see Eq. (3.1)), to maximize the revenue the channel members would also increase prices. The increment rate of $\hat{p}_{r, c}^{\mathrm{NI}}$ is higher than that of $\hat{p}_{d, c}^{\mathrm{NI}}$ suggests that the reference price has a stronger impact on $\hat{p}_{r, c}^{\mathrm{NI}}$ than on $\hat{p}_{d, c}^{\mathrm{NI}}$, which is straightforwardly attributed to the assumption that the reference price affects the demand comes from the retail channel directly. The last item implies that the more pessimistic the manufacturer is to the market, the smaller the prices she will charge, which is consistent with the fact. When the market size is relatively small, higher prices would lose more customers, which in turn would lose more revenue.

\subsection{Decentralized DCSC}

In this part, we explore the optimal pricing decisions in a decentralized DCSC, where the channel members aim to maximize their individual revenues by setting their own prices. Specifically, we define $w_{d}^{\mathrm{NI}}, p_{r, d}^{\mathrm{NI}}$ and $p_{d, d}^{\mathrm{NI}}$ respectively as the wholesale, retail, direct sale prices; $\Pi_{m, d}^{\mathrm{NI}}$ and $\Pi_{r, d}^{\mathrm{NI}}$ as the revenues of the manufacturer and retailer, respectively.

The problem in the decentralized scenario can be formulated as a Stackelberg game. In such a game, the manufacturer first decides prices $w_{d}^{\mathrm{NI}}$ and $p_{d, d}^{\mathrm{NI}}$, then the retailer decides $p_{r, d}^{\mathrm{NI}}$ follows from the manufacturer's pricing decisions. We derive the optimal price $\hat{p}_{r, d}^{\mathrm{NI}}$ followed from the optimal prices $\hat{w}_{d}^{\mathrm{NI}}$ and $\hat{p}_{d, d}^{\mathrm{NI}}$, utilizing backward induction and two-stage optimization technique. 
Proposition 4.3. In the decentralized DCSC of NI case, $\mathbb{E}_{m, d}^{\mathrm{NI}}$ is jointly concave in $w_{d}^{\mathrm{NI}}$ and $p_{d, d}^{\mathrm{NI}}$; There exists two thresholds on reference price $\bar{r}_{d}^{\mathrm{NI}}=\frac{\theta a\left(3 b_{1} b_{2}+3 b_{2} \eta^{+}-\beta^{2}\right)+2(1-\theta) \tilde{a} \beta\left(b_{1}+\eta^{+}\right)}{4\left(b_{1} b_{2}+b_{2} \eta^{+}-\beta^{2}\right) b_{1}+\left(b_{1} b_{2}+b_{2} \eta^{+}-3 \beta^{2}\right) \eta^{+}}$and $\underline{r}_{d}^{\mathrm{NI}}=$ $\frac{\theta a\left(3 b_{1} b_{2}+3 b_{2} \eta^{-}-\beta^{2}\right)+2(1-\theta) \tilde{a} \beta\left(b_{1}+\eta^{-}\right)}{4\left(b_{1} b_{2}+b_{2} \eta^{-}-\beta^{2}\right) b_{1}+\left(b_{1} b_{2}+b_{2} \eta^{-}-3 \beta^{2}\right) \eta^{-}}, \bar{r}_{d}^{\mathrm{NI}} \geq \underline{r}_{d}^{\mathrm{NI}}$, such that

(i) if $r \geq \bar{r}_{d}^{\mathrm{NI}}$, the optimal pricing decisions are

$$
\begin{aligned}
& \hat{w}_{d}^{\mathrm{NI}}=\frac{1}{2} \frac{b_{2}\left(\theta a+\eta^{+} r\right)+\beta(1-\theta) \tilde{a}}{b_{1} b_{2}+b_{2} \eta^{+}-\beta^{2}}, \hat{p}_{d, d}^{\mathrm{NI}}=\frac{1}{2} \frac{\beta\left(\theta a+\eta^{+} r\right)+\left(b_{1}+\eta^{+}\right)(1-\theta) \tilde{a}}{b_{1} b_{2}+b_{2} \eta^{+}-\beta^{2}}, \\
& \hat{p}_{r, d}^{\mathrm{NI}}=\frac{\left(\theta a+\eta^{+} r\right)\left(3 b_{1} b_{2}+3 b_{2} \eta^{+}-\beta^{2}\right)}{4\left(b_{1} b_{2}+b_{2} \eta^{+}-\beta^{2}\right)\left(b_{1}+\eta^{+}\right)}+\frac{(1-\theta) \tilde{a} \beta}{2\left(b_{1} b_{2}+b_{2} \eta^{+}-\beta^{2}\right)} ;
\end{aligned}
$$

(ii) if $r \leq \underline{r}_{d}^{\mathrm{NI}}$, the optimal pricing decisions are

$$
\begin{aligned}
& \hat{w}_{d}^{\mathrm{NI}}=\frac{1}{2} \frac{b_{2}\left(\theta a+\eta^{-} r\right)+\beta(1-\theta) \tilde{a}}{b_{1} b_{2}+b_{2} \eta^{-}-\beta^{2}}, \hat{p}_{d, d}^{\mathrm{NI}}=\frac{1}{2} \frac{\beta\left(\theta a+\eta^{-} r\right)+\left(b_{1}+\eta^{-}\right)(1-\theta) \tilde{a}}{b_{1} b_{2}+b_{2} \eta^{-}-\beta^{2}}, \\
& \hat{p}_{r, d}^{\mathrm{NI}}=\frac{\left(\theta a+\eta^{-} r\right)\left(3 b_{1} b_{2}+3 b_{2} \eta^{-}-\beta^{2}\right)}{4\left(b_{1} b_{2}+b_{2} \eta^{-}-\beta^{2}\right)\left(b_{1}+\eta^{-}\right)}+\frac{(1-\theta) \tilde{a} \beta}{2\left(b_{1} b_{2}+b_{2} \eta^{-}-\beta^{2}\right)} ;
\end{aligned}
$$

(iii) if $\underline{r}_{d}^{\mathrm{NI}}<r<\bar{r}_{d}^{\mathrm{NI}}$, the optimal pricing decisions are

$$
\begin{aligned}
\hat{p}_{r, d}^{\mathrm{NI}} & =r, \hat{w}_{d}^{\mathrm{NI}}=\frac{4 b_{2}\left(\left(2 b_{1}+\eta\right) r-\theta a\right)-2(1-\theta) \tilde{a} \beta-\beta^{2} r}{4\left(\beta^{2}+2 b_{2}\left(b_{1}+\eta\right)\right)}, \\
\hat{p}_{d, d}^{\mathrm{NI}} & =\frac{\beta\left(5 b_{1} r+3 \eta r-2 \theta a\right)+2\left(b_{1}+\eta\right)(1-\theta) \tilde{a}}{2\left(\beta^{2}+2 b_{2}\left(b_{1}+\eta\right)\right.} .
\end{aligned}
$$

Besides, the manufacturer's and retailer's optimal expected revenues, $\mathbb{E} \hat{\Pi}_{m, d}^{\mathrm{NI}}$ and $\mathbb{E} \hat{\Pi}_{r, d}^{\mathrm{NI}}$, are given by equation (4.1) when $w^{\mathrm{NI}}, p_{r}^{\mathrm{NI}}$, and $p_{d}^{\mathrm{NI}}$ respectively equal to $\hat{w}_{d}^{\mathrm{NI}}, \hat{p}_{r, d}^{\mathrm{NI}}$, and $\hat{p}_{d, d}^{\mathrm{NI}}$.

Proposition 4.3 shows that the manufacturer's expected revenue is jointly concave in her pricing decisions, which indicates that $\hat{w}_{d}^{\mathrm{NI}}$ and $\hat{p}_{d, d}^{\mathrm{NI}}$ are the unique solution to maximize $\mathbb{E} \Pi_{m, d}^{\mathrm{NI}}$. Comparing the optimal prices in the centralized and decentralized DCSCs (see Props. 4.1 and 4.3), we discover that the optimal direct sale prices in the two scenarios are identical; the optimal retail price in the centralized scenario equals the optimal wholesale price in the decentralized scenario; the two thresholds on reference price in the two scenarios are different, which affects the optimal decisions significantly. If the reference price equals a value that between the two thresholds on reference price, we assume the optimal retail price is the reference price. Partial of these results are similar to Hua et al. [13], in which they did not consider reference price but considered the delivery lead time, which they believe greatly affects the customers' demands.

Proposition 4.4. (i) $\hat{p}_{r, d}^{\mathrm{NI}}$ is increasing in $r$; (ii) $\hat{w}_{d}^{\mathrm{NI}}$ and $\hat{p}_{d, d}^{\mathrm{NI}}$ are piecewise functions of $r$, and they are increasing in $r$ when $r \geq \bar{r}_{d}^{\mathrm{NI}}, r \leq \underline{r}_{d}^{\mathrm{NI}}$ and $\underline{r}_{d}^{\mathrm{NI}}<r<\bar{r}_{d}^{\mathrm{NI}}$, respectively. (iii) Both $\hat{w}_{d}^{\mathrm{NI}}$ and $\hat{p}_{d, d}^{\mathrm{NI}}$ would suddenly increase when $r$ beyond $\underline{r}_{d}^{\mathrm{NI}}$ but would suddenly decrease when $r$ beyond $\bar{r}_{d}^{\mathrm{NI}}$.

A higher reference price implies that the customers are willing to pay more for the products, which naturally leads the retailer to raise the retail price to increase his revenue. Similarly, the manufacturer would also increase her prices. Due to the asymmetric RE on the demand function, the optimal prices are piecewise function of the reference price, and there exists two thresholds on reference price. The last item can be attributed to the fact that the manufacturer would set her wholesale price large enough but no more than the retail price to maximize her personal revenue in the decentralized DCSC. 


\subsection{Model misspecification}

In this section, we further respectively analyze the influences of the asymmetry RE on the retailer's and the manufacturer's pricing decisions and revenues in the centralized and decentralized DCSCs. Specially, we assume the retailer ignores $\mathrm{RE}$ but it exists in the consumers' mind factually and inevitably affect their purchasing behaviors.

In the centralized DCSC, we define $\bar{p}_{r, c}^{\mathrm{NI}}$ and $\bar{p}_{d, c}^{\mathrm{NI}}$ as the optimal retail and direct sale prices if the retailer ignores RE (i.e., $\eta^{+}, \eta^{-}=0$ ). It follows from Proposition 4.1 that

$$
\bar{p}_{r, c}^{\mathrm{NI}}=\frac{1}{2} \frac{\theta a b_{2}+(1-\theta) \tilde{a} \beta}{b_{1} b_{2}-\beta^{2}} \text { and } \bar{p}_{d, c}^{\mathrm{NI}}=\frac{1}{2} \frac{\beta \theta a+(1-\theta) \tilde{a} b_{1}}{b_{1} b_{2}-\beta^{2}} .
$$

Corollary 4.5. In the centralized DCSC of NI case, the optimal prices that without RE are higher than those with RE, i.e., $\bar{p}_{r, c}^{\mathrm{NI}} \geq \hat{p}_{r, c}^{\mathrm{NI}}$ and $\bar{p}_{d, c}^{\mathrm{NI}} \geq \hat{p}_{d, c}^{\mathrm{NI}}$.

Corollary 4.5 immediately implies that the optimal prices would be increasing if RE is ignored, which can also be verified by Proposition 4.2(i). Moreover, if the retailer ignores RE, the demands from two channels that the channel members believe are

$$
\bar{D}_{r, c}^{\mathrm{NI}}=\theta a-b_{1} \bar{p}_{r, c}^{\mathrm{NI}}+\beta \bar{p}_{d, c}^{\mathrm{NI}} \text { and } \bar{D}_{d, c}^{\mathrm{NI}}=(1-\theta) \tilde{a}-b_{2} \bar{p}_{d, c}^{\mathrm{NI}}+\beta \bar{p}_{r, c}^{\mathrm{NI}} .
$$

However, since RE exists in the customers' minds factually and inevitably their purchasing behaviors, the actual demands would be

$$
\tilde{D}_{r, c}^{\mathrm{NI}}=\theta a-b_{1} \bar{p}_{r, c}^{\mathrm{NI}}+\beta \bar{p}_{d, c}^{\mathrm{NI}}+\eta\left(r-\bar{p}_{r, c}^{\mathrm{NI}}\right) \text { and } \tilde{D}_{d, c}^{\mathrm{NI}}=\bar{D}_{d, c}^{\mathrm{NI}} .
$$

Respectively define $\bar{\Pi}_{c}^{\mathrm{NI}}$ and $\tilde{\Pi}_{c}^{\mathrm{NI}}$ as the optimal expected revenues they expect to obtain and actually would obtain, then we have

$$
\mathbb{E} \bar{\Pi}_{c}^{\mathrm{NI}}=\bar{p}_{r, c}^{\mathrm{NI}} \bar{D}_{r, c}^{\mathrm{NI}}+\bar{p}_{d, c}^{\mathrm{NI}} \bar{D}_{d, c}^{\mathrm{NI}} \text { and } \mathbb{E} \tilde{\Pi}_{c}^{\mathrm{NI}}=\bar{p}_{r, c}^{\mathrm{NI}} \tilde{D}_{r, c}^{\mathrm{NI}}+\bar{p}_{d, c}^{\mathrm{NI}} \tilde{D}_{d, c}^{\mathrm{NI}}
$$

Proposition 4.6. In the centralized DCSC, (i) $\mathbb{E} \bar{\Pi}_{c}^{\mathrm{NI}}$ is independent of $r$; (ii) Both $\mathbb{E} \hat{\Pi}_{c}^{\mathrm{NI}}$ and $\mathbb{E} \tilde{\Pi}_{c}^{\mathrm{NI}}$ are increasing in $r$; (iii) $\mathbb{E} \bar{\Pi}_{c}^{\mathrm{NI}} \geq \mathbb{E} \tilde{\Pi}_{c}^{\mathrm{NI}}$ when $r \leq \bar{p}_{r, c}^{\mathrm{NI}}$ and $\mathbb{E}_{\bar{\Pi}}^{\mathrm{NI}}<\mathbb{E} \tilde{\Pi}_{c}^{\mathrm{NI}}$ when $r>\bar{p}_{r, c}^{\mathrm{NI}} ;$ (iv) $\mathbb{E} \hat{\Pi}_{c}^{\mathrm{NI}}>\mathbb{E} \tilde{\Pi}_{c}^{\mathrm{NI}}$.

If the retailer ignores $\mathrm{RE}$, the total revenue that the channel members expect to obtain is independent of the reference price, which can be attributed to the fact that the channel members' optimal prices and demands do not depend on the reference price if RE is ignored. However, the actual demands are determined by the customers and are increasing in the reference price, hence the actual revenue is increasing in the reference price.

The optimal revenue is increasing in the reference price is consistent with the actual situation. Specifically, customers with higher reference prices implies that they are willing to pay more for the products, which immediately increases the revenue. On the other hand, a relatively small reference price $\left(e . g ., r \leq \bar{p}_{r, c}^{\mathrm{NI}}\right)$ implies the customers in the market are negative about the market if the channel members ignore the fact, the total revenue they expected to obtain is generally higher than the actual total revenue they would obtain, i.e., they will overestimate the revenue, and vice versa. Furthermore, the optimal revenue the channel members are able to obtain is larger than the actual revenue they would obtain, which can be attributed to the fact that RE exists in the customers' mind factually, and the pricing decisions without RE are always suboptimal decisions.

In the decentralized DCSC, if the retailer ignores RE, it follows from Propositions 4.3 that the channel members would set $\bar{p}_{r, d}^{\mathrm{FI}}, \bar{w}_{d}^{\mathrm{FI}}$ and $\bar{p}_{d, c}^{\mathrm{FI}}$ as the optimal prices, where

$$
\bar{w}_{d}^{\mathrm{NI}}=\frac{1}{2} \frac{b_{2} \theta a+\beta(1-\theta) \tilde{a}}{b_{1} b_{2}-\beta^{2}}, \bar{p}_{d, d}^{\mathrm{NI}}=\frac{1}{2} \frac{\beta \theta a+b_{1}(1-\theta) \tilde{a}}{b_{1} b_{2}-\beta^{2}}, \bar{p}_{r, d}^{\mathrm{NI}}=\frac{\theta a\left(3 b_{1} b_{2}-\beta^{2}\right)+2(1-\theta) \tilde{a} \beta b_{1}}{4\left(b_{1} b_{2}-\beta^{2}\right) b_{1}},
$$


then the demands from the two channels they believe are

$$
\bar{D}_{r, d}^{\mathrm{NI}}=\theta a-b_{1} \bar{p}_{r, d}^{\mathrm{NI}}+\beta \bar{p}_{d, d}^{\mathrm{NI}} \text { and } \bar{D}_{d, d}^{\mathrm{NI}}=(1-\theta) \tilde{a}-b_{2} \bar{p}_{d, d}^{\mathrm{NI}}+\beta \bar{p}_{r, d}^{\mathrm{NI}} .
$$

However, because RE exists in the customers' minds factually and inevitably their purchasing behaviors, the actual demands they would obtain are

$$
\tilde{D}_{r, c}^{\mathrm{NI}}=\theta a-b_{1} \bar{p}_{r, c}^{\mathrm{NI}}+\beta \bar{p}_{d, c}^{\mathrm{NI}}+\eta\left(r-\bar{p}_{r, c}^{\mathrm{NI}}\right) \text { and } \tilde{D}_{d, c}^{\mathrm{NI}}=\bar{D}_{d, c}^{\mathrm{NI}} .
$$

The optimal revenue the manufacturer expects to obtain and she actually would obtain are

$$
\mathbb{E} \bar{\Pi}_{m, d}^{\mathrm{NI}}=\bar{w}_{d}^{\mathrm{NI}} \bar{D}_{r, d}^{\mathrm{NI}}+\bar{p}_{d, d}^{\mathrm{NI}} \bar{D}_{d, d}^{\mathrm{NI}} \text { and } \mathbb{E} \tilde{\Pi}_{m, d}^{\mathrm{NI}}=\bar{w}_{d}^{\mathrm{NI}} \tilde{D}_{r, d}^{\mathrm{NI}}+\bar{p}_{d, d}^{\mathrm{NI}} \tilde{D}_{d, d}^{\mathrm{NI}}
$$

Similarly, the optimal revenue the retailer expects to obtain and he actually would obtain are

$$
\mathbb{E} \bar{\Pi}_{r, d}^{\mathrm{NI}}=\left(\bar{p}_{r, d}^{\mathrm{NI}}-\bar{w}_{d}^{\mathrm{NI}}\right) \bar{D}_{r, d}^{\mathrm{NI}} \text { and } \mathbb{E} \tilde{\Pi}_{r, d}^{\mathrm{NI}}=\left(\bar{p}_{r, d}^{\mathrm{NI}}-\bar{w}_{d}^{\mathrm{NI}}\right) \tilde{D}_{r, d}^{\mathrm{NI}} .
$$

Proposition 4.7. When $r \leq \bar{p}_{r, d}^{\mathrm{NI}}, \mathbb{E} \bar{\Pi}_{m, d}^{\mathrm{NI}} \geq \mathbb{E} \tilde{\Pi}_{m, d}^{\mathrm{NI}}$ and $\mathbb{E} \bar{\Pi}_{r, d}^{\mathrm{NI}} \geq \mathbb{E} \tilde{\Pi}_{r, d}^{\mathrm{NI}} ;$ When $r>\bar{p}_{r, d}^{\mathrm{NI}}, \mathbb{E}_{m, d}^{\mathrm{NI}}<\mathbb{E} \tilde{\Pi}_{m, d}^{\mathrm{NI}}$ and $\mathbb{E} \bar{\Pi}_{r, d}^{\mathrm{NI}}<\mathbb{E} \tilde{\Pi}_{r, d}^{\mathrm{NI}}$.

It follows from Proposition 4.7 that a relatively small reference price implies that the customers take a pessimistic view of the market. If RE is ignored, the actual revenue that the channel members would obtain is smaller than the revenue they expect to obtain, and vice versa. The results are similar to Proposition 4.6. It is noted that in regard to the value of RE to the channel members, we will illustrate them in Section 6.

\section{INFORMATION SHARING (IS) CASE}

In this section, we consider a case where the retailer is willing to share the market information with the manufacturer, a contract exists between them: once the manufacturer accepts the information, she must pay the unit information cost $k$ to the retailer no matter which channels her demand comes from. Note that if the manufacturer only needs to pay the demand that from the retail channel, she would choose to sell products through the direct sale channel after receiving the information to avoid paying a high fee (especially when $k$ is large enough). Besides, it is reasonable to assume the unit information cost $k$ is not larger than the direct sale price and the wholesale price, i.e., $0<k \leq p_{d}^{\mathrm{IS}}$ and $0<k \leq w^{\mathrm{IS}}$; Otherwise, the manufacturer would reject the information, then the model would be the same as Section 4 .

To avoid confusing notation, we add the superscript "IS" to the notation showed in Section 3 to denote the corresponding notation in this section, add the subscript "c" (resp. "d") to denote the corresponding notation in the centralized (resp. decentralized) DCSC. Specifically, we define $w^{\mathrm{IS}}, p_{r}^{\mathrm{IS}}$ and $p_{d}^{\mathrm{IS}}$ as the pricing decisions; $D_{r}^{\mathrm{IS}}$ and $D_{d}^{\mathrm{IS}}$ as the demands; $\Pi_{m}^{\mathrm{IS}}$ and $\Pi_{r}^{\mathrm{IS}}$ as the manufacturer's and retailer's revenues, $\Pi^{\mathrm{IS}}$ as the total revenue of DCSC. Once the manufacturer accepts the market information, the demands from the two channels are

$$
D_{r}^{\mathrm{IS}}=\theta a-b_{1} p_{r}^{\mathrm{IS}}+\beta p_{d}^{\mathrm{IS}}+\eta\left(r-p_{r}^{\mathrm{IS}}\right) \text { and } D_{d}^{\mathrm{IS}}=(1-\theta) a-b_{2} p_{d}^{\mathrm{IS}}+\beta p_{r}^{\mathrm{IS}} .
$$

The manufacturer's and the retailer's revenues are

$$
\Pi_{m}^{\mathrm{IS}}=\left(w^{\mathrm{IS}}-k\right) D_{r}^{\mathrm{IS}}+\left(p_{d}^{\mathrm{IS}}-k\right) D_{d}^{\mathrm{IS}} \text { and } \Pi_{r}^{\mathrm{IS}}=\left(p_{r}^{\mathrm{IS}}-w^{\mathrm{IS}}\right) D_{r}^{\mathrm{IS}}+k\left(D_{r}^{\mathrm{IS}}+D_{d}^{\mathrm{IS}}\right) .
$$

The total revenue of DCSC is

$$
\Pi^{\mathrm{IS}}=\Pi_{m}^{\mathrm{IS}}+\Pi_{r}^{I S I}=p_{r}^{\mathrm{NI}} D_{r}^{\mathrm{IS}}+p_{d}^{\mathrm{IS}} D_{d}^{\mathrm{IS}} .
$$




\subsection{Centralized DCSC}

We utilize the same method to analyze the centralized DCSC in the NI case (see Sect. 4.1), the channel members optimize their own prices to maximize the revenue of the entire DCSC. Similarly, denote $p_{r, c}^{\mathrm{IS}}$ and $p_{d, c}^{\mathrm{IS}}$ as the pricing decisions, $\Pi_{c}^{\mathrm{IS}}$ as the revenue of the entire DCSC, then we obtain the following optimal pricing decisions.

Proposition 5.1. In the centralized DCSC of IS case, $\Pi_{c}^{\mathrm{IS}}$ is jointly concave in $p_{r, c}^{\mathrm{IS}}$ and $p_{d, c}^{\mathrm{IS}}$. There exists two thresholds on reference price $\bar{r}_{c}^{\mathrm{IS}}=\frac{\theta a b_{2}+(1-\theta) a \beta}{2 b_{1} b_{2}+\eta^{+} b_{2}-2 \beta^{2}} \geq \underline{r}_{c}^{\mathrm{IS}}=\frac{\theta a b_{2}+(1-\theta) a \beta}{2 b_{1} b_{2}+\eta^{-} b_{2}-2 \beta^{2}}$, such that

(i) if $r \geq \bar{r}_{c}^{\mathrm{IS}}$, the optimal prices are

$$
\hat{p}_{r, c}^{\mathrm{IS}}=\frac{1}{2} \frac{\theta a b_{2}+b_{2} \eta^{+} r+(1-\theta) a \beta}{\left(b_{1}+\eta^{+}\right) b_{2}-\beta^{2}} \text { and } \hat{p}_{d, c}^{\mathrm{IS}}=\frac{1}{2} \frac{\beta \theta a+\beta \eta^{+} r+(1-\theta) a\left(b_{1}+\eta^{+}\right)}{\left(b_{1}+\eta^{+}\right) b_{2}-\beta^{2}} ;
$$

(ii) if $r \leq \underline{r}_{c}^{\mathrm{IS}}$, the optimal prices are

$$
\hat{p}_{r, c}^{\mathrm{IS}}=\frac{1}{2} \frac{\theta a b_{2}+b_{2} \eta^{-} r+(1-\theta) a \beta}{\left(b_{1}+\eta^{-}\right) b_{2}-\beta^{2}} \text { and } \hat{p}_{d, c}^{\mathrm{IS}}=\frac{1}{2} \frac{\beta \theta a+\beta \eta^{-} r+(1-\theta) a\left(b_{1}+\eta^{-}\right)}{\left(b_{1}+\eta^{-}\right) b_{2}-\beta^{2}} ;
$$

(iii) otherwise, the optimal prices are

$$
\hat{p}_{r, c}^{\mathrm{IS}}=r \text { and } \hat{p}_{d, c}^{\mathrm{IS}}=\frac{(1-\theta) a+2 \beta r}{2 b_{2}} .
$$

Besides, the optimal revenue of the entire DCSC, $\hat{\Pi}_{c}^{\mathrm{IS}}$, is given by equation (5.2) when $p_{r}^{\mathrm{IS}}$ and $p_{d}^{\mathrm{IS}}$ respectively equal to $\hat{p}_{r, d}^{\mathrm{IS}}$ and $\hat{p}_{d, d}^{\mathrm{IS}}$.

Comparing to the optimal prices in the decentralized DCSCs that with and without IS (Props. 4.1 and 5.1), we uncover that the market information strongly influences the pricing decisions, specifically, a higher market size would lead to higher prices, while the information cost $k$ has no effects on them; the structures of the pricing decisions are similar, and the structure properties about $\hat{p}_{r, c}^{\mathrm{NI}}$ and $\hat{p}_{d, c}^{\mathrm{NI}}$ still hold for $\hat{p}_{r, c}^{\mathrm{IS}}$ and $\hat{p}_{d, c}^{\mathrm{IS}}$. We conclude the following results about $\hat{p}_{r, c}^{\mathrm{IS}}$ and $\hat{p}_{d, c}^{\mathrm{IS}}$.

Proposition 5.2. IS has no influence on the structures of the optimal prices in the centralized DCSC.

Utilizing the same method applied in Proposition 4.2, we obtain the similar results, we find that the higher the reference price, or the smaller the intensity of $\mathrm{RE}$, the higher the retail and direct sale prices, i.e., the optimal prices $\hat{p}_{r, c}^{\mathrm{IS}}$ and $\hat{p}_{d, c}^{\mathrm{IS}}$, are nonincreasing in $\eta^{+}$and $\eta^{-}$; increasing in $r$. Besides, we find that the increment rate of $\hat{p}_{r, c}^{\mathrm{IS}}$ is larger than that of $\hat{p}_{d, c}^{\mathrm{IS}}$. The results further implies that the structures of the optimal prices in the centralized scenario with IS is similar to that without IS.

\subsection{Decentralized DCSC}

We investigate the optimal pricing decisions in a decentralized DCSC with IS. Similar to the analysis in the decentralized DCSC with IS (see Sect. 4.2), the channel members aim to maximize their individual revenues by optimizing pricing decisions. Define $w_{d}^{\mathrm{IS}}, p_{r, d}^{\mathrm{IS}}$ and $p_{d, d}^{\mathrm{IS}}$ as the pricing decisions; $\Pi_{m, d}^{\mathrm{IS}}$ and $\Pi_{r, d}^{\mathrm{IS}}$ as the revenues generated by the manufacturer and the retailer. Formulate this problem as a Stackelberg game and utilize a two-stage optimization technique to solve it, then we can obtain the following results.

Proposition 5.3. In the decentralized DCSC of IS case, $\Pi_{m, d}^{\mathrm{IS}}$ is jointly concave in $w_{d}^{\mathrm{IS}}$ and $p_{d, d}^{\mathrm{IS}}$. There exists two thresholds on reference price $\bar{r}_{d}^{\mathrm{IS}} \geq \underline{r}_{d}^{\mathrm{IS}}$, where

$$
\bar{r}_{d}^{\mathrm{IS}}=\frac{\theta a\left(3 b_{1} b_{2}+3 b_{2} \eta^{+}-\beta^{2}\right)+2(1-\theta) a \beta\left(b_{1}+\eta^{+}\right)+2 k \beta\left(b_{1} b_{2}+b_{2} \eta^{+}-\beta^{2}\right)}{4\left(b_{1} b_{2}+b_{2} \eta^{+}-\beta^{2}\right) b_{1}+\left(b_{1} b_{2}+b_{2} \eta^{+}-3 \beta^{2}\right) \eta^{+}},
$$




$$
\underline{r}_{d}^{\mathrm{IS}}=\frac{\theta a\left(3 b_{1} b_{2}+3 b_{2} \eta^{-}-\beta^{2}\right)+2(1-\theta) a \beta\left(b_{1}+\eta^{-}\right)+2 k \beta\left(b_{1} b_{2}+b_{2} \eta^{-}-\beta^{2}\right)}{4\left(b_{1} b_{2}+b_{2} \eta^{-}-\beta^{2}\right) b_{1}+\left(b_{1} b_{2}+b_{2} \eta^{-}-3 \beta^{2}\right) \eta^{-}} .
$$

(i) If $r \geq \bar{r}_{d}^{\mathrm{IS}}$, the optimal prices are

$$
\begin{aligned}
\hat{w}_{d}^{\mathrm{IS}} & =\frac{1}{2} \frac{\left(\theta a+\eta^{+} r\right) b_{2}+\left[(1-\theta) a-b_{2} k\right] \beta}{b_{1} b_{2}+b_{2} \eta^{+}-\beta^{2}}+\frac{1}{2} \frac{\beta^{3} k}{\left(b_{1} b_{2}+b_{2} \eta^{+}-\beta^{2}\right)\left(b_{1}+\eta^{+}\right)}+k, \\
\hat{p}_{d, d}^{\mathrm{IS}} & =\frac{1}{2} \frac{\left(\theta a+\eta^{+} r\right) \beta+\left[(1-\theta) a+b_{2} k\right]\left(b_{1}+\eta^{+}\right)-\beta^{2} k}{b_{1} b_{2}+b_{2} \eta^{+}-\beta^{2}} \\
\hat{p}_{r, d}^{\mathrm{IS}} & =\frac{\left(\theta a+\eta^{+} r\right)\left(3 b_{1} b_{2}+3 b_{2} \eta^{+}-\beta^{2}\right)+2(1-\theta) a \beta\left(b_{1}+\eta^{+}\right)}{4\left(b_{1} b_{2}+b_{2} \eta^{+}-\beta^{2}\right)\left(b_{1}+\eta^{+}\right)}+\frac{k \beta}{2\left(b_{1}+\eta^{+}\right)} .
\end{aligned}
$$

(ii) If $r \leq \underline{r}_{d}^{\mathrm{IS}}$, the optimal prices are

$$
\begin{aligned}
\hat{w}_{d}^{\mathrm{IS}} & =\frac{1}{2} \frac{\left(\theta a+\eta^{-} r\right) b_{2}+\left[(1-\theta) a-b_{2} k\right] \beta}{b_{1} b_{2}+b_{2} \eta^{-}-\beta^{2}}+\frac{1}{2} \frac{\beta^{3} k}{\left(b_{1} b_{2}+b_{2} \eta^{-}-\beta^{2}\right)\left(b_{1}+\eta^{-}\right)}+k, \\
\hat{p}_{d, d}^{\mathrm{IS}} & =\frac{1}{2} \frac{\left(\theta a+\eta^{-} r\right) \beta+\left[(1-\theta) a+b_{2} k\right]\left(b_{1}+\eta^{-}\right)-\beta^{2} k}{b_{1} b_{2}+b_{2} \eta^{-}-\beta^{2}}, \\
\hat{p}_{r, d}^{\mathrm{IS}} & =\frac{\left(\theta a+\eta^{-} r\right)\left(3 b_{1} b_{2}+3 b_{2} \eta^{-}-\beta^{2}\right)+2(1-\theta) a \beta\left(b_{1}+\eta^{-}\right)}{4\left(b_{1} b_{2}+b_{2} \eta^{-}-\beta^{2}\right)\left(b_{1}+\eta^{-}\right)}+\frac{k \beta}{2\left(b_{1}+\eta^{-}\right)} .
\end{aligned}
$$

(iii) Otherwise, the optimal prices are

$$
\begin{aligned}
& \hat{p}_{r, d}^{\mathrm{IS}}=r, \hat{w}_{d}^{\mathrm{IS}}=\frac{2 b_{2}\left(b_{1} r-\theta a\right)-(1-\theta) a \beta-3 b_{2} k \beta}{2\left(\beta^{2}+b_{2}\left(b_{1}+\eta\right)\right)}-\frac{k \beta^{2}}{2\left(b_{1}+\eta\right)\left(\beta^{2}+b_{2}\left(b_{1}+\eta\right)\right)}+k, \\
& \hat{p}_{d, d}^{\mathrm{IS}}=\frac{2 \beta\left(\left(2 b_{1}+\eta\right) r-\theta a\right)+\left(b_{1}+\eta\right)\left((1-\theta) a+b_{2} k\right)-k \beta^{2}}{2\left(\beta^{2}+b_{2}\left(b_{1}+\eta\right)\right)} .
\end{aligned}
$$

Besides, the optimal revenues of the manufacturer and the retailer, $\hat{\Pi}_{m, d}^{\mathrm{IS}}$ and $\hat{\Pi}_{r, d}^{\mathrm{IS}}$ are given by equation (5.1) when $w^{\mathrm{IS}}, p_{r}^{\mathrm{IS}}$ and $p_{d}^{\mathrm{IS}}$ respectively equal to $\hat{w}_{d}^{\mathrm{IS}}, \hat{p}_{r, d}^{\mathrm{IS}}$, and $\hat{p}_{d, d}^{\mathrm{IS}}$.

By analyzing the optimal pricing decisions in Proposition 5.3, we obtain the following structure properties, which are similar to Proposition 4.4.

Corollary 5.4. (i) $\hat{p}_{r, d}^{\mathrm{IS}}$ is increasing in $r$; (ii) $\hat{w}_{d}^{\mathrm{IS}}$ and $\hat{p}_{d, d}^{\mathrm{IS}}$ are piecewise functions of $r$, they are increasing in $r$ when $r \geq \bar{r}_{d}^{\mathrm{IS}}, r \leq \underline{r}_{d}^{\mathrm{IS}}$ and $\underline{r}_{d}^{\mathrm{IS}}<r<\bar{r}_{d}^{\mathrm{IS}}$, respectively. (iii) Both $\hat{w}_{d}^{\mathrm{IS}}$ and $\hat{p}_{d, d}^{\mathrm{IS}}$ would suddenly increase when $r$ beyond $\underline{r}_{d}^{\mathrm{IS}}$ but would suddenly decrease when $r$ beyond $\bar{r}_{d}^{\mathrm{IS}}$.

Comparing the results in Corollary 5.4 and Proposition 4.4, we uncover that in the decentralized DCSC, whether the information is shared or not, the monotonicity of the optimal prices is unchanged. However, IS greatly influences the thresholds on reference price, which implies that given a reference price $r$, the optimal prices in IS and NI cases are quite different. Therefore, one significant managerial insight derived from Corollary 5.4 and Proposition 4.4 is that the channel members should jointly consider the asymmetric RE and IS before making pricing decisions.

Proposition 5.5. In the decentralized DCSC of IS case, given a reference price $r$, the optimal prices are nondecreasing in $k$. Specially, the increment rate of $\hat{w}_{d}^{\mathrm{IS}}$ is not less than that of $\hat{p}_{d, d}^{\mathrm{IS}}$, which in turn is not less than that of $\hat{p}_{r, d}^{\mathrm{IS}}$. 
Comparing the optimal prices in the centralized and decentralized DCSCs of IS case, i.e., Propositions 5.1 and 5.5, we discover that the information cost $k$ only affects the optimal pricing decisions in the decentralized DCSC, and the optimal prices are increasing in $k$. This is consistent with the fact. As the increasing of the information cost $k$, the manufacturer would increase $\hat{w}_{d}^{\mathrm{IS}}$ and $\hat{p}_{d, d}^{\mathrm{IS}}$ naturally to maximize her personal revenue. From the retailer's perspective, as the wholesale price increases, he would also correspondingly increase the retail price to avoid losing revenue. The manufacturer must pay $k$ to the retailer once she sells out a product, thus it would be more eager for the manufacturer to increase her prices than the retailer to increase his price as $k$ increases.

\subsection{Model misspecification}

In this part, we further analyze the influences of the asymmetry RE on the channel members' pricing decisions and revenues if the retailer is willing to share the market information with the manufacturer. A similar analysis method to Section 4.3 is applied. That is, we first derive the optimal pricing decisions and revenues that the channel members believed if the retailer ignores $\mathrm{RE}$, however, $\mathrm{RE}$ exists in the consumers' minds really and inevitably affects their behaviors. Then, we compare the optimal solutions they believed and the actually obtained to illustrate the influences of the asymmetry RE. In the following, we respectively analyze the model in the centralized and decentralized DCSCs.

In the centralized DCSC, if the retailer ignores RE, $\bar{p}_{r, c}^{\mathrm{IS}}$ and $\bar{p}_{d, c}^{\mathrm{IS}}$ would be set as the optimal prices. It follows from Proposition 5.1 that

$$
\bar{p}_{r, c}^{\mathrm{IS}}=\frac{1}{2} \frac{\theta a b_{2}+(1-\theta) a \beta}{b_{1} b_{2}-\beta^{2}} \text { and } \bar{p}_{d, c}^{\mathrm{IS}}=\frac{1}{2} \frac{\beta \theta a+(1-\theta) a b_{1}}{b_{1} b_{2}-\beta^{2}} .
$$

Corollary 5.6. In the centralized DCSC of IS case, the optimal prices that without RE are higher than those with $R E$, i.e., $\bar{p}_{r, c}^{\mathrm{IS}} \geq \hat{p}_{r, c}^{\mathrm{IS}}$ and $\bar{p}_{d, c}^{\mathrm{IS}} \geq \hat{p}_{d, c}^{\mathrm{IS}}$.

The results in Corollary 5.6 are similar to the results in Corollary 4.5. If $\bar{p}_{r, c}^{\mathrm{IS}}$ and $\bar{p}_{d, c}^{\mathrm{IS}}$ are set as the optimal prices, the demands of the two channels that the channel members believe are

$$
\bar{D}_{r, c}^{\mathrm{IS}}=\theta a-b_{1} \bar{p}_{r, c}^{\mathrm{IS}}+\beta \bar{p}_{d, c}^{\mathrm{NI}} \text { and } \bar{D}_{d, c}^{\mathrm{IS}}=(1-\theta) a-b_{2} \bar{p}_{d, c}^{\mathrm{NI}}+\beta \bar{p}_{r, c}^{\mathrm{NI}} .
$$

Due to the reality existence of RE, the actual demands they would obtain are

$$
\tilde{D}_{r, c}^{\mathrm{IS}}=\theta a-b_{1} \bar{p}_{r, c}^{\mathrm{NI}}+\beta \bar{p}_{d, c}^{\mathrm{NI}}+\eta\left(r-\bar{p}_{r, c}^{\mathrm{NI}}\right) \text { and } \tilde{D}_{d, c}^{\mathrm{IS}}=\bar{D}_{d, c}^{\mathrm{NI}}
$$

Denote $\bar{\Pi}_{c}^{\mathrm{NI}}$ and $\tilde{\Pi}_{c}^{\mathrm{NI}}$ as the optimal total revenue they expect to obtain and they actually would obtain, then

$$
\bar{\Pi}_{c}^{\mathrm{IS}}=\bar{p}_{r, c}^{\mathrm{IS}} \bar{D}_{r, c}^{\mathrm{IS}}+\bar{p}_{d, c}^{\mathrm{IS}} \bar{D}_{d, c}^{\mathrm{IS}} \text { and } \tilde{\Pi}_{c}^{\mathrm{IS}}=\bar{p}_{r, c}^{\mathrm{IS}} \tilde{D}_{r, c}^{\mathrm{IS}}+\bar{p}_{d, c}^{\mathrm{IS}} \tilde{D}_{d, c}^{\mathrm{IS}}
$$

Proposition 5.7. (i) $\bar{\Pi}_{c}^{\mathrm{IS}}$ is independent of $r$; (ii) Both $\hat{\Pi}_{c}^{\mathrm{IS}}$ and $\tilde{\Pi}_{c}^{\mathrm{IS}}$ are increasing in $r$; (ii) $\bar{\Pi}_{c}^{\mathrm{IS}} \geq \tilde{\Pi}_{c}^{\mathrm{IS}}$ when $r \leq \bar{p}_{r, c}^{\mathrm{IS}}$ and $\bar{\Pi}_{c}^{\mathrm{IS}}<\tilde{\Pi}_{c}^{\mathrm{IS}}$ when $r>\bar{p}_{r, c}^{\mathrm{IS}} ;($ iv $) \hat{\Pi}_{c}^{\mathrm{IS}}>\tilde{\Pi}_{c}^{\mathrm{IS}}$.

The results shown in Proposition 5.7 are similar to Proposition 4.6. It is worth noting that both the optimal prices and demand functions are linear in the market size whether the information is shared or not, and the revenue is the product of the optimal prices and demand. Hence, the structural properties of the revenues in the two scenarios are similar apparently.

In the decentralized DCSC, if the retailer ignores RE, the channel members would set the optimal prices as follows:

$$
\bar{w}_{d}^{\mathrm{IS}}=\frac{1}{2} \frac{\theta a b_{2}+\left[(1-\theta) a-b_{2} k\right] \beta}{b_{1} b_{2}-\beta^{2}}+\frac{1}{2} \frac{\beta^{3} k}{\left(b_{1} b_{2}-\beta^{2}\right) b_{1}}+k
$$




$$
\bar{p}_{d, d}^{\mathrm{IS}}=\frac{1}{2} \frac{\theta a \beta+\left[(1-\theta) a+b_{2} k\right] b_{1}-\beta^{2} k}{b_{1} b_{2}-\beta^{2}}, \bar{p}_{r, d}^{\mathrm{IS}}=\frac{\theta a\left(3 b_{1} b_{2}-\beta^{2}\right)+2(1-\theta) a \beta b_{1}}{4\left(b_{1} b_{2}-\beta^{2}\right) b_{1}}+\frac{k \beta}{2 b_{1}},
$$

and the demands of the two channels that they believed are

$$
\bar{D}_{r, d}^{\mathrm{IS}}=\theta a-b_{1} \bar{p}_{r, d}^{\mathrm{IS}}+\beta \bar{p}_{d, d}^{\mathrm{IS}} \text { and } \bar{D}_{d, d}^{\mathrm{IS}}=(1-\theta) a-b_{2} \bar{p}_{d, d}^{\mathrm{IS}}+\beta \bar{p}_{r, d}^{\mathrm{IS}} .
$$

Due to the reality existence of RE, the actual demands they would obtain are

$$
\tilde{D}_{r, c}^{\mathrm{IS}}=\theta a-b_{1} \bar{p}_{r, c}^{\mathrm{IS}}+\beta \bar{p}_{d, c}^{\mathrm{IS}}+\eta\left(r-\bar{p}_{r, c}^{\mathrm{IS}}\right) \text { and } \tilde{D}_{d, c}^{\mathrm{IS}}=\bar{D}_{d, c}^{\mathrm{IS}}
$$

Denote $\bar{\Pi}_{m, d}^{\mathrm{IS}}\left(\right.$ resp. $\left.\bar{\Pi}_{r, d}^{\mathrm{IS}}\right)$ and $\tilde{\Pi}_{m, d}^{\mathrm{IS}}\left(\right.$ resp. $\left.\tilde{\Pi}_{r, d}^{\mathrm{IS}}\right)$ respectively as the optimal revenues the manufacturer(resp. retailer) expects to obtain and she(resp. he) actually would obtain. Then,

$$
\begin{aligned}
& \bar{\Pi}_{m, d}^{\mathrm{IS}}=\left(\bar{w}_{d}^{\mathrm{IS}}-k\right) \bar{D}_{r, d}^{\mathrm{IS}}+\left(\bar{p}_{d, d}^{\mathrm{IS}}-k\right) \bar{D}_{d, d}^{\mathrm{IS}}, \bar{\Pi}_{r, d}^{\mathrm{IS}}=\left(\bar{p}_{r, d}^{\mathrm{IS}}-\bar{w}_{d}^{\mathrm{IS}}\right) \bar{D}_{r, d}^{\mathrm{IS}}+k\left(\bar{D}_{r, d}^{\mathrm{IS}}+\bar{D}_{d, d}^{\mathrm{IS}}\right) ; \\
& \tilde{\Pi}_{m, d}^{\mathrm{IS}}=\left(\bar{w}_{d}^{\mathrm{IS}}-k\right) \tilde{D}_{r, d}^{\mathrm{IS}}+\left(\bar{p}_{d, d}^{\mathrm{IS}}-k\right) \tilde{D}_{d, d}^{\mathrm{IS}}, \tilde{\Pi}_{r, d}^{\mathrm{IS}}=\left(\bar{p}_{r, d}^{\mathrm{IS}}-\bar{w}_{d}^{\mathrm{IS}}\right) \tilde{D}_{r, d}^{\mathrm{IS}}+k\left(\tilde{D}_{r, d}^{\mathrm{IS}}+\tilde{D}_{d, d}^{\mathrm{IS}}\right) .
\end{aligned}
$$

Proposition 5.8. Given a reference price $r, \hat{\Pi}_{m, d}^{\mathrm{IS}}, \tilde{\Pi}_{m, d}^{\mathrm{IS}}$ and $\bar{\Pi}_{m, d}^{\mathrm{IS}}$ are decreasing in $k$; while $\hat{\Pi}_{r, d}^{\mathrm{IS}}$, $\tilde{\Pi}_{r, d}^{\mathrm{IS}}$ and $\bar{\Pi}_{r, d}^{\mathrm{IS}}$ are increasing in $k$.

Proposition 5.8 suggests whether RE is ignored or not, as the increase of the information cost $k$, the manufacturer's revenue would decrease, while the retailer's revenue would increase. These results are apparently consistent with the fact: As $k$ increases, the manufacturer would pay more for the retailer once she sells out a product. Similar to the analysis of the revenues that the channel members expect to obtain and they actually would obtain, in the decentralized DCSC of NI case, we obtain the following results.

Corollary 5.9. When $r \leq \bar{p}_{r, d}^{\mathrm{IS}}$, $\bar{\Pi}_{r, d}^{\mathrm{IS}} \geq \tilde{\Pi}_{r, d}^{\mathrm{IS}}$ and $\bar{\Pi}_{m, d}^{\mathrm{IS}} \geq \tilde{\Pi}_{m, d}^{\mathrm{IS}}$; When $r<\bar{p}_{r, d}^{\mathrm{IS}}$, $\bar{\Pi}_{r, d}^{\mathrm{IS}}<\tilde{\Pi}_{r, d}^{\mathrm{IS}}$ and $\bar{\Pi}_{m, d}^{\mathrm{IS}}<\tilde{\Pi}_{m, d}^{\mathrm{IS}}$.

By analyzing the optimal prices and the corresponding revenues in Sections 4 and 5, several interesting questions are raised: (i) how much revenue will be lost in the centralized (or decentralized) DCSC if RE is ignored? Especially when the actual reference price is relatively large or small. (ii) Whether IS is always better for the entire DCSC and the channel members than non-information sharing? (iii) Compared with the IS case, how much revenue will be increased (or decreased) for the entire DCSC and the channel members, if the information is shared between the channel members?

In the following, we will illustrate the above questions by numerical experiments.

\section{The VAlue of the ASymmetric RE}

We implement numerical experiments to examine the value of the asymmetric RE on the channel members' pricing decisions and the corresponding revenues in the centralized and decentralized DCSCs with and without IS, respectively. We evaluate the performance of RE to the retailer by numerically comparing the optimal revenue she is able to obtain, the revenue she anticipated to obtain, and the revenue she actually would obtain, when she ignores RE that exists among the customers in reality, in the cases with and without IS. Clearly, the larger the deviations between the three types of revenues, the greater the influence of RE on the retailer, which further suggests the significance of the retailer's correct cognition on RE. Our numerical experiments have the following basic parameters: $a_{\mathrm{L}}=2, a_{\mathrm{H}}=8, \theta=0.4 ; b_{1}=4, b_{2}=3, \beta=2, \eta_{1}=0.5, \eta_{2}=1.8$. 


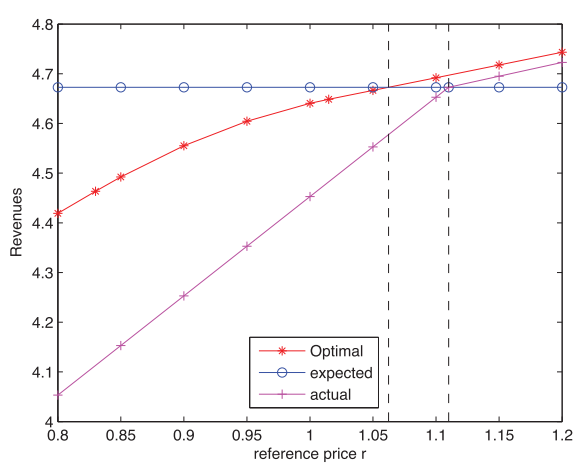

(a)

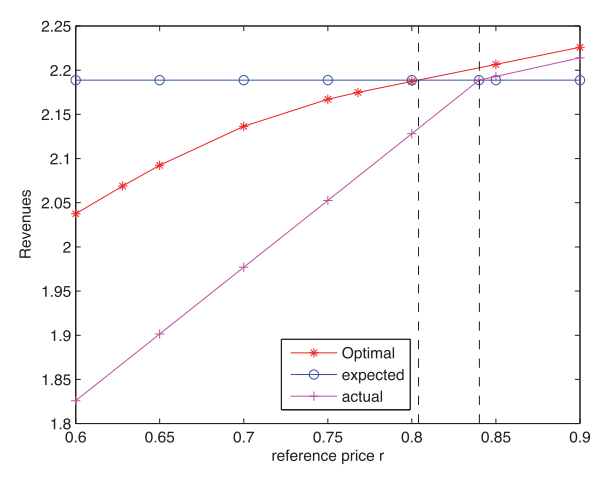

(b)

Figure 2. (a) Revenues in the centralized scenario when $\lambda=0.2$. (b) Revenues in the centralized scenario when $\lambda=0.8$.

\subsection{NI case}

Here we examine the value of $\mathrm{RE}$ to the retailer in the NI case, where the manufacturer deems the market size equals $a_{\mathrm{L}}$ with probability $\lambda$ and $a_{\mathrm{H}}$ with $1-\lambda$. Clearly, $\lambda$ has nonnegligible impacts on the three types of revenues, and we illustrate the impacts by comparing the revenues when $\lambda=0.2$ and $\lambda=0.8$, in the centralized and decentralized cases, respectively.

Centralized DCSC: observing Figures 2a and 2b, if the retailer ignores RE, both the optimal revenue she could be able to obtain and the actual revenue she would obtain are increasing in $r$ but decreasing in $\lambda$. These are consistent with the theoretical results shown in Section 4.1. Another phenomenon we observed is that the optimal revenues are always larger than the actual revenue, especially when the reference price is small enough, which suggests that ignoring the asymmetric RE would lose much revenue, and $\mathrm{RE}$ has a positive effect on the retailer. Besides, a larger $\lambda$ gives rise to two smaller thresholds on reference price, which further implies that ignoring RE will lose more revenue for DCSC with a larger $\lambda$.

Define

$$
\frac{\mid \text { the optimal revenue }- \text { the actual revenue } \mid}{\text { the actual revenue }} * 100 \%
$$

as the revenue loss rate if $\mathrm{RE}$ is ignored, we uncover that as the actual reference price $r=0.8$, the loss rate is $9.14 \%$ when $\lambda=0.2$, and is $2.35 \%$ when $\lambda=0.8$.

Decentralized DCSC: from Figure 3, we observed that the three types of revenues are increasing in $\lambda$. The channel members' revenues are fluctuating with the reference price, which is caused by the asymmetric RE. There exists two thresholds on reference prices, the revenues when a reference price equals a value that is between the two thresholds on reference prices are generally higher than those when the reference price equals a value that is outside of this interval. Besides, we discover that in the decentralized scenario, ignoring $\mathrm{RE}$ is always detrimental to the manufacturer, but may benefit the retailer especially when the actual reference price equals a value between the two thresholds on reference price.

\subsection{IS case}

Next, we explore the value of RE in the IS case, where the retailer shares the market information with the manufacturer, and then both of them know the market size clearly.

Centralized DCSC: comparing the three types of revenues in Figures 2 and 4, we found that in the centralized scenario, sharing information will increase two thresholds on reference price, which suggests that ignoring RE 


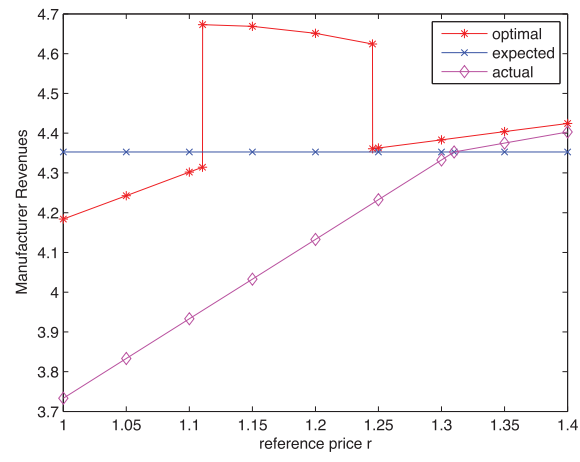

(a)

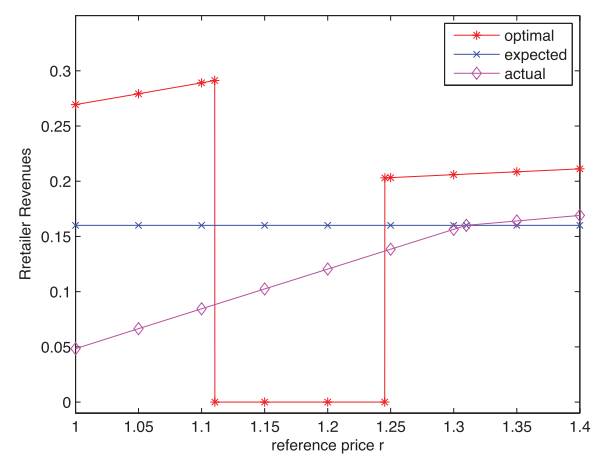

(c)

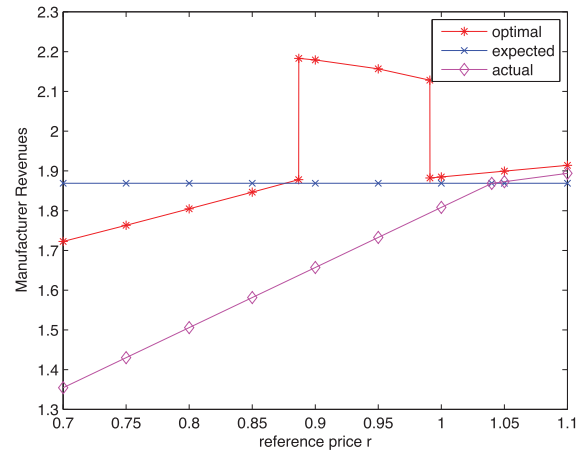

(b)

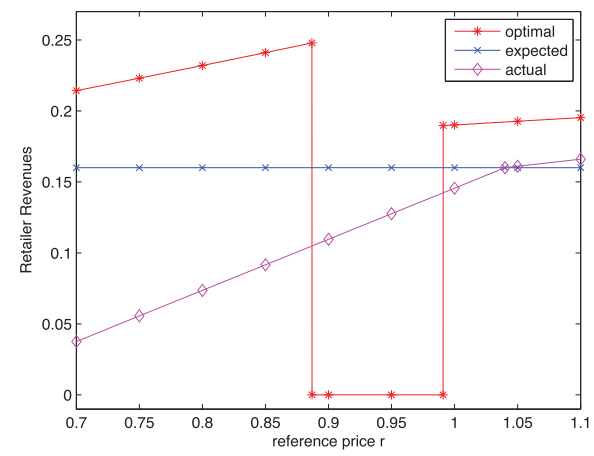

(d)

Figure 3. (a), (b) Manufacturer's revenues in the decentralized DCSC; (c), (d) Retailer's revenues in the decentralized DCSC. (a) $\lambda=0.2$. (b) $\lambda=0.8$. (c) $\lambda=0.2$. (d) $\lambda=0.8$.

will lose more revenue when the information is shared than not shared, especially when $\lambda$ is large. For example, when $\lambda=0.8$ and $r=0.8$, the loss rate is $2.35 \%$ in the NI case, whereas is $9.2 \%$ in the IS case.

Decentralized DCSC: comparing Figures 3 and 5, a higher $k$ generally gives rise to higher revenue for the retailer but a lower revenue for the manufacturer; Moreover, we found that as $k$ increases, ignoring RE will lose more revenue for the manufacturer, especially when the reference price is relatively small. For example, when $r=1.1$ the loss rate is $10.9 \%$ when $k=0.05$, but $16.7 \%$ when $k=0.35$. However, as $k$ increases, ignoring RE would benefit the retailer, excluding the case where the actual reference price is between the two thresholds on reference prices. For example, if $r=1.1$ the loss rate is $150 \%$ when $k=0.05$, but $43 \%$ when $k=0.35$. Another interesting insight we obtain is that whatever $k$ is, ignoring the reference price is always detrimental to the channel members but may benefit the retailer when $k$ is small enough and the actual reference price is between the two thresholds on reference price.

Besides, it follows from Figures 2-5 we observe that whether the information is shared or not, the channel members generally underestimate revenues under a relatively high reference price, while overestimating the revenues under a relatively small reference price, in either centralized DCSC or decentralized DCSC, 


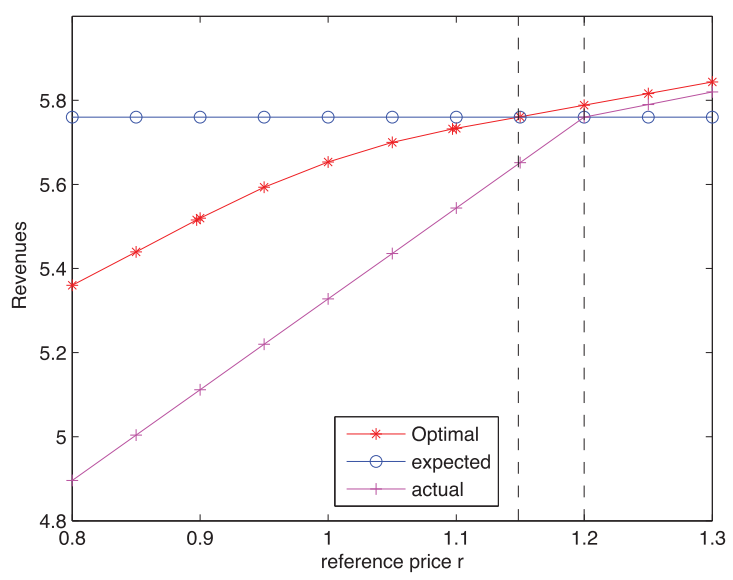

FigURE 4. Revenues in centralized DCSC of the IS case.

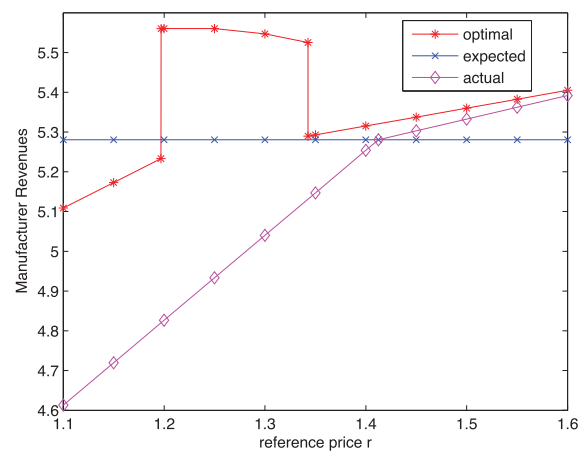

(a)

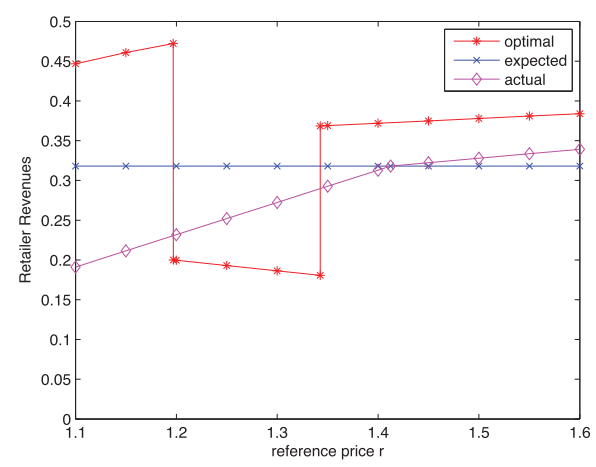

(c)

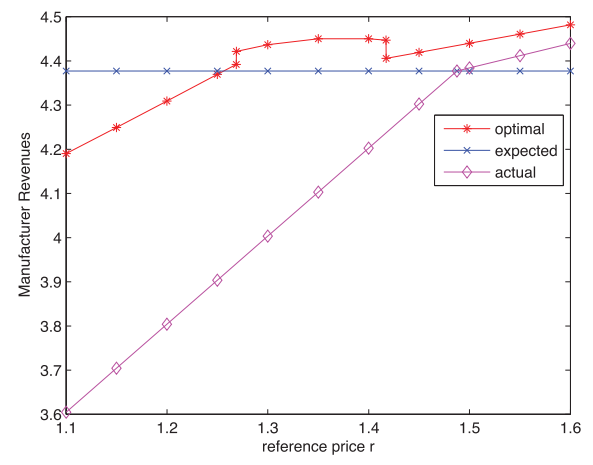

(b)

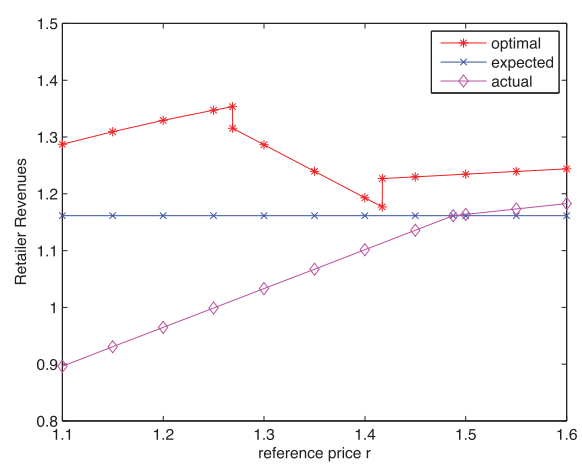

(d)

Figure 5. (a), (b) Manufacturer's revenues in the decentralized DCSC; (c), (d) Retailer's revenues in the decentralized DCSC. (a) $k=0.05$. (b) $k=0.35$. (c) $k=0.05$. (d) $k=0.35$. 
TABLE 2. The value of the information to DCSC.

\begin{tabular}{llllllllllll}
\hline \hline \multicolumn{10}{c}{$V_{c}$} \\
\hline \multirow{2}{*}{$\lambda$} & 0.6 & 0.65 & 0.7 & 0.75 & 0.8 & 0.85 & 0.9 & 0.95 & 1 & 1.05 & 1.1 \\
\hline \multirow{nyyyyyyyyyyy}{0}{0.2} & 0.9218 & 0.9266 & 0.9315 & 0.9363 & 0.9411 & 0.9471 & 0.9648 & 0.9888 & 1.0128 & 1.0335 & 1.0413 \\
0.8 & 3.0141 & 3.0348 & 3.0675 & 3.1144 & 3.1728 & 3.2330 & 3.2939 & 3.3477 & 3.3881 & 3.4151 & 3.4287 \\
\hline
\end{tabular}

TABLE 3. The value of the information to the manufacturer.

\begin{tabular}{llllllllllll}
\hline \hline \multicolumn{10}{c}{$V_{m, d}$} \\
\cline { 2 - 11 }$k$ & 1 & 1.05 & 1.1 & 1.15 & 1.2 & 1.25 & 1.3 & 1.35 & 1.4 & 1.45 & 1.5 \\
\hline 0.05 & 0.7032 & 0.7068 & 0.7104 & 0.4071 & 0.4884 & 1.0819 & 1.0521 & 1.0088 & 0.7948 & 0.7962 & 0.7977 \\
0.35 & -0.1957 & -0.1967 & -0.1978 & -0.5058 & -0.4292 & -0.0807 & -0.0565 & -0.0598 & -0.0764 & -0.1126 & -0.1128 \\
\hline
\end{tabular}

TABLE 4 . The value of the information to the retailer.

\begin{tabular}{llllllllllll}
\hline \hline \multicolumn{10}{c}{$V_{r, d}$} \\
\cline { 2 - 12 }$k$ & 1 & 1.05 & 1.1 & 1.15 & 1.2 & 1.25 & 1.3 & 1.35 & 1.4 & 1.45 & 1.5 \\
\hline \multirow{nyyyyyyyyyyy}{0.05}{} & 0.2536 & 0.2548 & 0.2561 & 0.5566 & 0.5681 & 0.1051 & 0.0918 & 0.0784 & 0.2546 & 0.2550 & 0.2555 \\
0.35 & 1.0728 & 1.0787 & 1.0846 & 1.3897 & 1.4059 & 1.2191 & 1.1888 & 1.1355 & 1.0821 & 1.0910 & 1.0931 \\
\hline
\end{tabular}

\section{The VAlue OF INFORMATion SHARING}

In this section, we numerically examine the value of IS, by comparing the three types of revenues in the centralized and decentralized DCSCs with and without IS, respectively.

We first evaluate the value in the centralized scenario. Clearly, comparing Figures 2 and 4, we can find the revenue of the entire DCSC would significantly improve with IS. In the following, we quantify the value of sharing information by separately comparing the revenues in the centralized and decentralized DCSCs when the market information is shared and not shared. Following from Yue and Liu [32], we use the relative gaps

$$
V_{c}=\hat{\Pi}_{c}^{\mathrm{IS}}-\hat{\Pi}_{c}^{\mathrm{NI}}, V_{m, d}=\hat{\Pi}_{m, d}^{\mathrm{IS}}-\hat{\Pi}_{m, d}^{\mathrm{NI}}, \text { and } V_{r, d}=\hat{\Pi}_{r, d}^{\mathrm{IS}}-\hat{\Pi}_{r, d}^{\mathrm{NI}}
$$

to denote the values of IS to the centralized DCSC, as well as the manufacturer and the retailer in the decentralized DCSC, respectively. Clearly, larger values of $V_{c}, V_{m, d}$ and $V_{r, d}$ implies greater values of IS.

Table 2 shows the value of IS to the centralized DCSC. Reference price $r$ varies from 0.6 to 1.1, and $\lambda \in$ $\{0.2,0.8\}$. We observe that the larger $\lambda$ is, the greater the value of IS is, which is consistent with the fact situation. A higher $\lambda$ suggests that the manufacturer is more negative about the market, and sharing the market information with her will bring higher revenue for the system. Furthermore, we find that a higher reference price will make IS more valuable to DCSC.

In Tables 3 and 4, we respectively illustrate the values of information sharing to the retailer and to the manufacturer in the decentralized DCSC. Taking $\lambda=0.2$, from Table 3, if $k$ is relatively small, accepting the market information would increase the manufacturer's revenue; Otherwise, accepting the market information would decrease her revenue, that is, it is better for the manufacturer to refuse IS. From Table 4 we observe that the retailer's revenue is increasing in the information cost $k$. A higher $k$ implies that the retailer will get 
more revenue for the manufacturer. One may suspect that the manufacturer could set a lower price for the direct sale channel to stimulate demand after accepting the information, whereas a contract regulates that the manufacturer must pay unit cost $k$ to the retailer no matter which channels her demand comes from. Hence, we conclude that the manufacturer tends to accept the market information from the retailer when $k$ is not large enough, while the retailer always supports IS.

\section{Conclusions}

Recently, ARE and IS are increasingly getting the attention of the dual-channel members, since both of them separately affect the customers' demand significantly. In this paper, we explore the joint effect of these two factors on a DCSC that consists of a retail and a direct sale channel. We first respectively explore the pricing decisions in a centralized and a decentralized DCSC with and without IS under the asymmetric RE, using the Stackelberg game and two-stage optimization technique. Then we evaluate the values of the asymmetric RE and IS to DCSC by introducing model misspecification and numerical experiments. Several interesting and important managerial insights are obtained. First, we find that a higher reference price or a weaker intensity of RE induces the channel members to increase prices, and make IS more valuable to them. Second, ignoring the asymmetric RE will lose more revenue when the information is shared than not shared, especially when the channel members take a pessimistic view of the market. Third, whether the information is shared or not, channel members generally underestimate revenues under a relatively high reference price, while overestimating the revenues under a relatively low reference price. Finally, the manufacturer conditionally accepts IS (i.e., when the information purchase cost is relatively small), while the retailer always accepts IS since sharing information brings him a windfall.

Several interesting and related topics are worthy of studying in the future. First, we assume the reference prices are evolved just from the retail prices. What are the corresponding results if the reference prices are evolved from the direct sale prices? In practice, customers may also evolve a reference price from the direct sale prices, but it may be more complicated. Second, we assume all customers in the market are risk-averse for the sake of analysis, but in reality, customers may be gain-seeking or risk-neutral. What happens to the system if the customers in the market are gain-seeking or risk-neutral, or a mixture of the above three types?

\section{Appendix A.}

Proof of Proposition 4.1. Taking the partial derivatives of $\Pi_{c}^{\mathrm{NI}}$ with respect to (w.r.t) $p_{r, c}^{\mathrm{NI}}$ and $p_{d, c}^{\mathrm{NI}}$, then we obtain the following Hessian matrix

$$
\mathrm{H}^{\mathrm{NI}}=\left(\begin{array}{cc}
\frac{\partial^{2} \Pi_{c}^{\mathrm{NI}}}{\partial\left(p_{r, c}^{\mathrm{NI}}\right)^{2}} & \frac{\partial^{2} \Pi_{c}^{\mathrm{NI}}}{\partial\left(p_{r, c}^{\mathrm{NI}}\right) \partial\left(p_{d, c}^{\mathrm{NI}}\right)} \\
\frac{\partial^{2} \Pi_{c}^{\mathrm{NI}}}{\partial\left(p_{r, c}^{\mathrm{NI}}\right) \partial\left(p_{d, c}^{\mathrm{NI}}\right)} & \frac{\partial^{2} \Pi_{c}^{\mathrm{NI}}}{\partial\left(p_{d, c}^{\mathrm{NI}}\right)^{2}}
\end{array}\right)=\left(\begin{array}{cc}
-2 b_{1}-2 \eta & 2 \beta \\
2 \beta & -2 b_{2}
\end{array}\right)
$$

Since $\frac{\partial^{2} \Pi_{c}^{\mathrm{NI}}}{\partial\left(p_{r, c}^{\mathrm{NI}}\right)^{2}}=-2 b_{1}-2 \eta<0$, and $\operatorname{det}\left(\mathrm{H}^{\mathrm{NI}}\right)=4\left(b_{1} b_{2}+b_{2} \eta-\beta^{2}\right)>0$ (where the inequalities follow from $b_{1}, b_{2}>\beta>0$ and $\left.\eta \in\left\{\eta^{+}, \eta^{-}\right\}>0\right)$, hence $\Pi_{c}^{\mathrm{NI}}$ is jointly concave in $p_{r, c}^{\mathrm{NI}}$ and $p_{d, c}^{\mathrm{NI}}$, which implies there exists a unique solution to maximize $\Pi_{c}^{\mathrm{NI}}$. Taking the derivatives of $\Pi_{c}^{\mathrm{NI}}$ w.r.t. $p_{r, c}^{\mathrm{FI}}$ and $p_{d, c}^{\mathrm{FI}}$, we obtain

$$
\frac{\partial \Pi_{c}^{\mathrm{NI}}}{\partial\left(p_{r, c}^{\mathrm{NI}}\right)}=\theta a-2\left(b_{1}+\eta\right) p_{r, c}^{\mathrm{NI}}+2 \beta p_{d, c}^{\mathrm{NI}}+\eta r \text { and } \frac{\partial \Pi_{c}^{\mathrm{NI}}}{\partial\left(p_{d, c}^{\mathrm{NI}}\right)}=(1-\theta) \tilde{a}+2 \beta p_{r, c}^{\mathrm{NI}}-2 b_{2} p_{d, c}^{\mathrm{NI}} .
$$

Setting the above formulae to zero, we obtain the optimal retail price and direct sale price

$$
\hat{p}_{r, c}^{\mathrm{NI}}=\frac{1}{2} \frac{\theta a b_{2}+b_{2} \eta r+(1-\theta) \tilde{a} \beta}{\left(b_{1}+\eta\right) b_{2}-\beta^{2}} \text { and } \hat{p}_{d, c}^{\mathrm{NI}}=\frac{1}{2} \frac{\beta \theta a+\beta \eta r+(1-\theta) \tilde{a}\left(b_{1}+\eta\right)}{\left(b_{1}+\eta\right) b_{2}-\beta^{2}} .
$$


Define $\bar{r}_{c}^{\mathrm{NI}}=\frac{\theta a b_{2}+(1-\theta) \tilde{a} \beta}{2 b_{1} b_{2}+\eta^{+} b_{2}-2 \beta^{2}}$ and $\underline{r}_{c}^{\mathrm{NI}}=\frac{\theta a b_{2}+(1-\theta) \tilde{a} \beta}{2 b_{1} b_{2}+\eta^{-} b_{2}-2 \beta^{2}}\left(\bar{r}_{c}^{\mathrm{NI}}>\underline{r}_{c}^{\mathrm{NI}}\right.$ since $\left.\eta^{-}>\eta^{+}\right)$. If the observed reference price is larger than the optimal retail price, then $\eta=\eta^{+}$, otherwise $\eta=\eta^{-}$, which implies that if $r \geq$ $\frac{1}{2} \frac{\theta a b_{2}+b_{2} \eta^{+} r+(1-\theta) \tilde{a} \beta}{\left(b_{1}+\eta^{+}\right) b_{2}-\beta^{2}}$, i.e., $r \geq \bar{r}_{c}^{\mathrm{NI}}$, the optimal retail price $\hat{p}_{r, c}^{\mathrm{NI}}=\frac{1}{2} \frac{\theta a b_{2}+b_{2} \eta^{+} r+(1-\theta) \tilde{a} \beta}{\left(b_{1}+\eta^{+}\right) b_{2}-\beta^{2}}$ and the optimal direct sale price $\hat{p}_{d, c}^{\mathrm{NI}}=\frac{1}{2} \frac{\beta \theta a+\beta \eta^{+} r+(1-\theta) \tilde{a}\left(b_{1}+\eta^{+}\right)}{\left(b_{1}+\eta^{+}\right) b_{2}-\beta^{2}}$

If $r \leq \frac{1}{2} \frac{\theta a b_{2}+b_{2} \eta^{-} r+(1-\theta) \tilde{a} \beta}{\left(b_{1}+\eta^{-}\right) b_{2}-\beta^{2}}$, i.e., $r \leq \underline{r}_{c}^{\mathrm{NI}}$, the optimal retail price $\hat{p}_{r, c}^{\mathrm{NI}} r=\frac{1}{2} \frac{\theta a b_{2}+b_{2} \eta^{-} r+(1-\theta) \tilde{a} \beta}{\left(b_{1}+\eta^{-}\right) b_{2}-\beta^{2}}$ and the optimal direct sale price $\hat{p}_{d, c}^{\mathrm{NI}}=\frac{1}{2} \frac{\beta \theta a+\beta \eta^{-} r+(1-\theta) \tilde{a}\left(b_{1}+\eta^{-}\right)}{\left(b_{1}+\eta^{-}\right) b_{2}-\beta^{2}}$;

Otherwise, if $\underline{r}_{c}^{\mathrm{NI}}<r<\bar{r}_{c}^{\mathrm{NI}}$, the optimal retail price $\hat{p}_{r, c}^{\mathrm{NI}}=r$. Substituting $\hat{p}_{r, c}^{\mathrm{NI}}=r$ into equation $(4.2)$ and maximizing the revenue $\Pi_{c}^{\mathrm{NI}}$, we obtain $\hat{p}_{d, c}^{\mathrm{NI}}=\frac{(1-\theta) \tilde{a}+2 \beta r}{2 b_{2}}$. Replacing $p_{r}^{\mathrm{NI}}$ and $p_{d}^{\mathrm{NI}}$ with $\hat{p}_{r, c}^{\mathrm{NI}}$ and $\hat{p}_{d, c}^{\mathrm{NI}}$ in equation (4.2), we obtain the optimal revenue of the entire DCSC.

Proof of Proposition 4.2. Since

$$
\begin{aligned}
& \frac{1}{2} \frac{\theta a b_{2}+b_{2} \eta^{+} \bar{r}_{c}^{\mathrm{NI}}+(1-\theta) \tilde{a} \beta}{\left(b_{1}+\eta^{+}\right) b_{2}-\beta^{2}}=\bar{r}_{c}^{\mathrm{NI}}, \frac{1}{2} \frac{\beta \theta a+\beta \eta^{+} \bar{r}_{c}^{\mathrm{NI}}+(1-\theta) \tilde{a}\left(b_{1}+\eta^{+}\right)}{\left(b_{1}+\eta^{+}\right) b_{2}-\beta^{2}}=\frac{(1-\theta) \tilde{a}+2 \beta \bar{r}_{c}^{\mathrm{NI}}}{2 b_{2}} \\
& \frac{1}{2} \frac{\theta a b_{2}+b_{2} \eta^{-} \underline{r}_{c}^{\mathrm{NI}}+(1-\theta) \tilde{a} \beta}{\left(b_{1}+\eta^{-}\right) b_{2}-\beta^{2}}=\underline{r}_{c}^{\mathrm{NI}}, \frac{1}{2} \frac{\beta \theta a+\beta \eta^{-} \underline{r}_{c}^{\mathrm{NI}}+(1-\theta) \tilde{a}\left(b_{1}+\eta^{-}\right)}{\left(b_{1}+\eta^{-}\right) b_{2}-\beta^{2}}=\frac{(1-\theta) \tilde{a}+2 \beta \underline{r}_{c}^{\mathrm{NI}}}{2 b_{2}} .
\end{aligned}
$$

Thus, we obtain that $\hat{p}_{r, c}^{\mathrm{NI}}$ and $\hat{p}_{d, c}^{\mathrm{NI}}$ are continuous in $r$. Taking the derivations of $\hat{p}_{r, c}^{\mathrm{NI}}$ and $\hat{p}_{d, c}^{\mathrm{NI}}$ w.r.t. $\eta^{+}$and $\eta^{-}$,

(i) if $r \geq \bar{r}_{c}^{\mathrm{NI}}$, we obtain that

$$
\frac{\partial \hat{p}_{r, c}^{\mathrm{NI}}}{\partial \eta^{+}}=\frac{b_{2} r\left(b_{1} b_{2}-\beta^{2}\right)-\theta a b_{2}^{2}-(1-\theta) \tilde{a} \beta b_{2}}{2\left(\left(b_{1}+\eta^{+}\right) b_{2}-\beta^{2}\right)^{2}}<0 \text { and } \frac{\partial \hat{p}_{d, c}^{\mathrm{NI}}}{\partial \eta^{+}}=\frac{\beta r\left(b_{1} b_{2}-\beta^{2}\right)-\theta a b_{2} \beta-(1-\theta) \tilde{a} \beta^{2}}{2\left(\left(b_{1}+\eta^{+}\right) b_{2}-\beta^{2}\right)^{2}}<0,
$$

where the inequalities follow from $r_{\max } \leq{\frac{\theta a b_{2}+(1-\theta) \tilde{a} \beta^{2}}{b_{1} b_{2}-\beta}}^{2}$ (see the analysis in Sect. 3).

(ii) If $r \leq \underline{r}_{c}^{\mathrm{NI}}$, we have

$$
\frac{\partial \hat{p}_{r, c}^{\mathrm{NI}}}{\partial \eta^{-}}=\frac{b_{2} r\left(b_{1} b_{2}-\beta^{2}\right)-\theta a b_{2}^{2}-(1-\theta) \tilde{a} \beta b_{2}}{2\left(\left(b_{1}+\eta^{-}\right) b_{2}-\beta^{2}\right)^{2}}<0 \text { and } \frac{\partial \hat{p}_{d, c}^{\mathrm{NI}}}{\partial \eta^{-}}=\frac{\beta r\left(b_{1} b_{2}-\beta^{2}\right)-\theta a b_{2} \beta-(1-\theta) \tilde{a} \beta^{2}}{2\left(\left(b_{1}+\eta^{-}\right) b_{2}-\beta^{2}\right)^{2}}<0 .
$$

(iii) If $\underline{r}_{c}^{\mathrm{NI}}<r<\bar{r}_{c}^{\mathrm{NI}}$,

$$
\frac{\partial \hat{p}_{r, c}^{\mathrm{NI}}}{\partial \eta^{-}}=\frac{\partial \hat{p}_{d, c}^{\mathrm{NI}}}{\partial \eta^{-}}=0
$$

Clearly, both $\hat{p}_{r, c}^{\mathrm{NI}}$ and $\hat{p}_{d, c}^{\mathrm{NI}}$ are nonincreasing in $\eta^{+}$and $\eta^{-}$.

In the following, we prove that $\hat{p}_{r, c}^{\mathrm{NI}}$ and $\hat{p}_{d, c}^{\mathrm{NI}}$ are increasing in $r$. It is apparently follows from Proposition 4.1 that $\hat{p}_{r, c}^{\mathrm{NI}}$ and $\hat{p}_{d, c}^{\mathrm{NI}}$ are increasing in $r$ when $r \geq \bar{r}_{c}^{\mathrm{NI}}, r \leq \underline{r}_{c}^{\mathrm{NI}}$ and $\underline{r}_{c}^{\mathrm{NI}}<r<\bar{r}_{c}^{\mathrm{NI}}$, respectively. Because $\hat{p}_{r, c}^{\mathrm{NI}}$ and $\hat{p}_{d, c}^{\mathrm{NI}}$ are continuous, thus $\hat{p}_{r, c}^{\mathrm{NI}}$ and $\hat{p}_{d, c}^{\mathrm{NI}}$ are increasing in $r$. Besides, when $r \geq \bar{r}_{c}^{\mathrm{NI}}$ or $r \leq \underline{r}_{c}^{\mathrm{NI}}, \frac{\partial \hat{p}_{r, c}^{\mathrm{NI}}}{\partial r}=\frac{b_{2} \eta^{ \pm}}{2\left[\left(b_{1}+\eta^{ \pm}\right) b_{2}-\beta^{2}\right]}>$ $\frac{\partial \hat{p}_{d, c}^{\mathrm{NI}}}{\partial r}=\frac{\beta \eta^{ \pm}}{2\left[\left(b_{1}+\eta^{ \pm}\right) b_{2}-\beta^{2}\right]}$; when $\underline{r}_{c}^{\mathrm{NI}}<r<\bar{r}_{c}^{\mathrm{NI}}, \frac{\partial \hat{p}_{r, c}^{\mathrm{NI}}}{\partial r}=1>\frac{\partial \hat{p}_{d, c}^{\mathrm{NI}}}{\partial r}=\frac{\beta}{b_{2}}$. Hence, we conclude that $\frac{\partial \hat{p}_{r, c}^{\mathrm{NI}}}{\partial r}>\frac{\partial \hat{p}_{d, c}^{\mathrm{NI}}}{\partial r}$ for any $r$.

Finally, we show the optimal prices are decreasing in $\lambda$. It immediately follows from the Proposition 4.1 that the optimal prices are increasing in $a$. Combining $\tilde{a}$ is decreasing in $\lambda\left(\tilde{a}=\lambda a_{\mathrm{L}}+(1-\lambda) a_{\mathrm{H}}\right)$, we obtain the optimal prices are decreasing in $\lambda$. 
Proof of Proposition 4.3. Given the manufacturer's decisions $p_{d, d}^{\mathrm{NI}}$ and $w_{d}^{\mathrm{NI}}$, the optimal retail price is given by deriving the first-order derivative of $\Pi_{r, d}^{\mathrm{NI}}$ w.r.t. $p_{r, d}^{\mathrm{NI}}$, then we obtain

$$
p_{r, d}^{\mathrm{NI}}=\frac{1}{2} \frac{\theta a+\eta r+\beta p_{d, d}^{\mathrm{NI}}}{b_{1}+\eta}+w_{d}^{\mathrm{NI}} .
$$

Substituting $p_{r, d}^{\mathrm{NI}}$ into equation (4.1), we obtain the manufacturer's revenue is

$$
\Pi_{m, d}^{\mathrm{NI}}=w_{d}^{\mathrm{NI}}\left[\theta a-b_{1} p_{r, d}^{\mathrm{NI}}+\beta p_{d, d}^{\mathrm{NI}}+\eta\left(r-p_{r, d}^{\mathrm{NI}}\right)\right]+p_{d, d}^{\mathrm{NI}}\left[(1-\theta) \tilde{a}-b_{2} p_{d, d}^{\mathrm{NI}}+\beta p_{r, d}^{\mathrm{NI}}\right] .
$$

Taking the derivatives of $\Pi_{m, d}^{\mathrm{NI}}$ w.r.t. $w_{d}^{\mathrm{NI}}$ and $p_{d, d}^{\mathrm{NI}}$, we get the following Hessian matrix

$$
\tilde{\mathrm{H}}^{\mathrm{NI}}=\left(\begin{array}{cc}
\frac{\partial^{2} \Pi_{m, d}^{\mathrm{NI}}}{\partial\left(w_{d}^{\mathrm{NI}}\right)^{2}} & \frac{\partial^{2} \Pi_{m, d}^{\mathrm{NI}}}{\partial\left(w_{d}^{\mathrm{NI}}\right) \partial\left(p_{d, d}^{\mathrm{NI}}\right)} \\
\frac{\partial^{2} \Pi_{m, d}^{\mathrm{NI}}}{\partial\left(p_{d, d}^{\mathrm{NI}}\right) \partial\left(w_{d}^{\mathrm{NI}}\right)} & \frac{\partial^{2} \Pi_{m, d}^{\mathrm{NI}}}{\partial\left(p_{d, d}^{\mathrm{NI}}\right)^{2}}
\end{array}\right)=\left(\begin{array}{cc}
-b_{1}-\eta & \beta \\
\beta & \frac{\beta^{2}}{b_{1}+\eta}-2 b_{2}
\end{array}\right) .
$$

Since $\frac{\partial^{2} \Pi_{m, d}^{\mathrm{NI}}}{\partial\left(w_{d}^{\mathrm{NI}}\right)^{2}}=-b_{1}-\eta<0$ and $\operatorname{det}\left(\tilde{\mathrm{H}}^{\mathrm{NI}}\right)=2\left(b_{1}+\eta\right) b_{2}-2 \beta^{2}>0$, hence $\Pi_{m, d}^{\mathrm{NI}}$ is jointly concave in $w_{d}^{\mathrm{NI}}$ and $p_{d, d}^{\mathrm{NI}}$, i.e., $\Pi_{m, d}^{\mathrm{NI}}$ has a unique solution. Computing the first-order derivatives of $\Pi_{m, d}^{\mathrm{NI}}$ w.r.t. $w_{d}^{\mathrm{NI}}$ and $p_{d, d}^{\mathrm{NI}}$

$$
\begin{aligned}
& \frac{\partial \Pi_{m, d}^{\mathrm{NI}}}{\partial w_{d}^{\mathrm{NI}}}=\frac{1}{2} \theta a+\frac{1}{2} \eta r+\beta p_{d, d}^{\mathrm{NI}}-b_{1} w_{d}^{\mathrm{NI}}-\eta w_{d}^{\mathrm{NI}}, \\
& \frac{\partial \Pi_{m, d}^{\mathrm{NI}}}{\partial p_{d, d}^{\mathrm{NI}}}=\beta w_{d}^{\mathrm{NI}}+\left(\frac{\beta^{2}}{b_{1}+\eta}-2 b_{2}\right) p_{d, d}^{\mathrm{NI}}+(1-\theta) \tilde{a}+\frac{\beta \theta a}{2\left(b_{1}+\eta\right)}+\frac{\beta \eta r}{2\left(b_{1}+\eta\right)} .
\end{aligned}
$$

Setting the above formulae to zero, the optimal retail price and optimal direct sale price are

$$
\hat{w}_{d}^{\mathrm{NI}}=\frac{b_{2} \theta a+\beta(1-\theta) \tilde{a}+b_{2} \eta r}{2\left(b_{1} b_{2}+b_{2} \eta-\beta^{2}\right)} \text { and } \hat{p}_{d, d}^{\mathrm{NI}}=\frac{1}{2} \frac{\beta \theta a+\left(b_{1}+\eta\right)(1-\theta) \tilde{a}+\beta \eta r}{b_{1} b_{2}+b_{2} \eta-\beta^{2}} .
$$

Substituting $\hat{w}_{d}^{\mathrm{NI}}$ and $\hat{p}_{d, d}^{\mathrm{NI}}$ into equation (A.2), we get $\hat{p}_{r, d}^{\mathrm{NI}}=\frac{(\theta a+\eta r)\left(3 b_{1} b_{2}+3 b_{2} \eta-\beta^{2}\right)}{4\left(b_{1} b_{2}+b_{2} \eta-\beta^{2}\right)\left(b_{1}+\eta\right)}+\frac{(1-\theta) \tilde{a} \beta}{2\left(b_{1} b_{2}+b_{2} \eta-\beta^{2}\right)}$.

Let $r=\hat{p}_{r, d}^{\mathrm{NI}}$, i.e., $r=\frac{\theta a\left(3 b_{1} b_{2}+3 b_{2} \eta-\beta^{2}\right)+2(1-\theta) \tilde{a} \beta\left(b_{1}+\eta\right)}{4\left(b_{1} b_{2}+b_{2} \eta-\beta^{2}\right) b_{1}+\left(b_{1} b_{2}+b_{2} \eta-3 \beta^{2}\right) \eta}, f\left(\eta^{ \pm}\right)=\frac{\theta a\left(3 b_{1} b_{2}+3 b_{2} \eta^{ \pm}-\beta^{2}\right)+2(1-\theta) \tilde{a} \beta\left(b_{1}+\eta^{ \pm}\right)}{4\left(b_{1} b_{2}+b_{2} \eta^{ \pm}-\beta^{2}\right) b_{1}+\left(b_{1} b_{2}+b_{2} \eta^{ \pm}-3 \beta^{2}\right) \eta^{ \pm}}$. Because

$$
\frac{\partial f\left(\eta^{ \pm}\right)}{\partial \eta^{ \pm}}=\frac{-b_{1}\left(b_{1} b_{2}+2 b_{2} \eta^{ \pm}+\beta^{2}\right)-b_{2}\left(\eta^{ \pm}\right)^{2}-\theta a b_{2}\left(b_{1}+\eta^{ \pm}\right)\left(3 b_{1} b_{2}+3 b_{2} \eta^{ \pm}-2 \beta^{2}\right)-3 \theta a \beta^{4}}{\left(4\left(b_{1} b_{2}+b_{2} \eta^{ \pm}-\beta^{2}\right) b_{1}+\left(b_{1} b_{2}+b_{2} \eta^{ \pm}-3 \beta^{2}\right) \eta^{ \pm}\right)^{2}}<0,
$$

and thus $f\left(\eta^{+}\right)>f\left(\eta^{-}\right)$. Define $\bar{r}_{d}^{\mathrm{NI}}=f\left(\eta^{+}\right)$and $\underline{r}_{d}^{\mathrm{NI}}=f\left(\eta^{-}\right)$.

Then we have: if $r \geq \frac{\theta a\left(3 b_{1} b_{2}+3 b_{2} \eta^{+}-\beta^{2}\right)+2(1-\theta) \tilde{a} \beta\left(b_{1}+\eta^{+}\right)}{4\left(b_{1} b_{2}+b_{2} \eta^{+}-\beta^{2}\right) b_{1}+\left(b_{1} b_{2}+b_{2} \eta^{+}-3 \beta^{2}\right) \eta^{+}}$, i.e., $r \geq \bar{r}_{d}^{\mathrm{NI}}$ the optimal prices are

$$
\begin{aligned}
& \hat{w}_{d}^{\mathrm{NI}}=\frac{1}{2} \frac{b_{2} \theta a+\beta(1-\theta) \tilde{a}+b_{2} \eta^{+} r}{b_{1} b_{2}+b_{2} \eta^{+}-\beta^{2}}, \hat{p}_{d, d}^{\mathrm{NI}}=\frac{1}{2} \frac{\beta \theta a+\left(b_{1}+\eta^{+}\right)(1-\theta) \tilde{a}+\beta \eta^{+} r}{b_{1} b_{2}+b_{2} \eta^{+}-\beta^{2}}, \\
& \hat{p}_{r, d}^{\mathrm{NI}}=\frac{\left(\theta a+\eta^{+} r\right)\left(3 b_{1} b_{2}+3 b_{2} \eta^{+}-\beta^{2}\right)}{4\left(b_{1} b_{2}+b_{2} \eta^{+}-\beta^{2}\right)\left(b_{1}+\eta^{+}\right)}+\frac{(1-\theta) \tilde{a} \beta}{2\left(b_{1} b_{2}+b_{2} \eta^{+}-\beta^{2}\right)} .
\end{aligned}
$$

If $r \leq \frac{\theta a\left(3 b_{1} b_{2}+3 b_{2} \eta^{-}-\beta^{2}\right)+2(1-\theta) \tilde{a} \beta\left(b_{1}+\eta^{-}\right)}{4\left(b_{1} b_{2}+b_{2} \eta^{-}-\beta^{2}\right) b_{1}+\left(b_{1} b_{2}+b_{2} \eta^{-}-3 \beta^{2}\right) \eta^{-}}$, i.e., $r \leq \underline{r}_{d}^{\mathrm{NI}}$, the optimal prices are

$$
\hat{w}_{d}^{\mathrm{NI}}=\frac{1}{2} \frac{b_{2} \theta a+\beta(1-\theta) \tilde{a}+b_{2} \eta^{-} r}{b_{1} b_{2}+b_{2} \eta^{-}-\beta^{2}}, \hat{p}_{d, d}^{\mathrm{NI}}=\frac{1}{2} \frac{\beta \theta a+\left(b_{1}+\eta^{-}\right)(1-\theta) \tilde{a}+\beta \eta^{-} r}{b_{1} b_{2}+b_{2} \eta^{-}-\beta^{2}},
$$




$$
\hat{p}_{r, d}^{\mathrm{NI}}=\frac{\left(\theta a+\eta^{-} r\right)\left(3 b_{1} b_{2}+3 b_{2} \eta^{-}-\beta^{2}\right)}{4\left(b_{1} b_{2}+b_{2} \eta^{-}-\beta^{2}\right)\left(b_{1}+\eta^{-}\right)}+\frac{(1-\theta) \tilde{a} \beta}{2\left(b_{1} b_{2}+b_{2} \eta^{-}-\beta^{2}\right)} .
$$

Otherwise, the optimal retail price would be $\hat{p}_{r, d}^{\mathrm{NI}}=r$. Then, the revenue of the manufacturer can be written as

$$
\Pi_{m, d}^{\mathrm{NI}}=w_{d}^{\mathrm{FI}}\left[\theta a-b_{1} r+\beta p_{d, d}^{\mathrm{NI}}\right]+p_{d, d}^{\mathrm{NI}}\left[(1-\theta) \tilde{a}-b_{2} p_{d, d}^{\mathrm{NI}}+\beta r\right] .
$$

Then, it follows from equation (A.2) that

$$
w_{d}^{\mathrm{NI}}=r-\frac{1}{2} \frac{\theta a+\eta r+\beta p_{d, d}^{\mathrm{NI}}}{b_{1}+\eta} .
$$

Substituting equation (A.4) into equation (A.3), and by maximizing equation (A.3) we obtain

$$
\hat{w}_{d}^{\mathrm{NI}}=\frac{4 b_{2}\left(\left(2 b_{1}+\eta\right) r-\theta a\right)-2(1-\theta) \tilde{a} \beta-\beta^{2} r}{4\left(\beta^{2}+2 b_{2}\left(b_{1}+\eta\right)\right)}, \hat{p}_{d, d}^{\mathrm{NI}}=\frac{\beta\left(5 b_{1} r+3 \eta r-2 \theta a\right)+2\left(b_{1}+\eta\right)(1-\theta) \tilde{a}}{2\left(\beta^{2}+2 b_{2}\left(b_{1}+\eta\right)\right)} .
$$

Proof of Proposition 4.4. Because

$$
\begin{aligned}
& \frac{\left(\theta a+\eta^{+} \bar{r}_{d}^{\mathrm{NI}}\right)\left(3 b_{1} b_{2}+3 b_{2} \eta^{+}-\beta^{2}\right)}{4\left(b_{1} b_{2}+b_{2} \eta^{+}-\beta^{2}\right)\left(b_{1}+\eta^{+}\right)}+\frac{(1-\theta) \tilde{a} \beta}{2\left(b_{1} b_{2}+b_{2} \eta^{+}-\beta^{2}\right)}=\bar{r}_{d}^{\mathrm{NI}}, \\
& \frac{\left(\theta a+\eta^{-} \underline{r}_{d}^{\mathrm{NI}}\right)\left(3 b_{1} b_{2}+3 b_{2} \eta^{-}-\beta^{2}\right)}{4\left(b_{1} b_{2}+b_{2} \eta^{-}-\beta^{2}\right)\left(b_{1}+\eta^{-}\right)}+\frac{(1-\theta) \tilde{a} \beta}{2\left(b_{1} b_{2}+b_{2} \eta^{-}-\beta^{2}\right)}=\underline{r}_{d}^{\mathrm{NI}},
\end{aligned}
$$

and thus $\hat{p}_{r, d}^{\mathrm{NI}}$ is continuous. Observing the optimal pricing decisions in Proposition 4.3, $\hat{p}_{r, d}^{\mathrm{NI}}$, $\hat{w}_{d}^{\mathrm{NI}}$ and $\hat{p}_{d, d}^{\mathrm{NI}}$ are increasing in $r$ when $r \geq \bar{r}_{d}^{\mathrm{NI}}, r \leq \underline{r}_{d}^{\mathrm{NI}}$ and $\underline{r}_{d}^{\mathrm{NI}}<r<\bar{r}_{d}^{\mathrm{NI}}$, respectively. Combining $\hat{p}_{r, d}^{\mathrm{NI}}$ is continuous, then $\hat{p}_{r, d}^{\mathrm{NI}}$ is increasing in $r$. In regard to the third part of Proposition 4.4. Let

$$
\hat{w}_{d}^{\mathrm{NI}}\left(\bar{r}_{d}^{\mathrm{NI}}\right)=\frac{1}{2} \frac{b_{2}\left(\theta a+\eta^{+} \bar{r}_{d}^{\mathrm{NI}}\right)+\beta(1-\theta) \tilde{a}}{b_{1} b_{2}+b_{2} \eta^{+}-\beta^{2}} \text { and } \hat{w}_{d}^{\mathrm{NI}}\left(\underline{r}_{d}^{\mathrm{NI}}\right)=\frac{1}{2} \frac{b_{2}\left(\theta a+\eta^{-} \underline{r}_{d}^{\mathrm{NI}}\right)+\beta(1-\theta) \tilde{a}}{b_{1} b_{2}+b_{2} \eta^{-}-\beta^{2}}
$$

as the optimal wholesale prices when $r=\bar{r}_{d}^{\mathrm{NI}}$ and $r=\underline{r}_{d}^{\mathrm{NI}}$, respectively;

$$
\hat{p}_{d, d}^{\mathrm{NI}}\left(\bar{r}_{d}^{\mathrm{NI}}\right)=\frac{1}{2} \frac{\beta\left(\theta a+\eta^{+} \bar{r}_{d}^{\mathrm{NI}}\right)+\left(b_{1}+\eta^{+}\right)(1-\theta) \tilde{a}}{b_{1} b_{2}+b_{2} \eta^{+}-\beta^{2}} \text { and } \hat{p}_{d, d}^{\mathrm{NI}}\left(\underline{r}_{d}^{\mathrm{NI}}\right)=\frac{1}{2} \frac{\beta\left(\theta a+\eta^{-} \underline{r}_{d}^{\mathrm{NI}}\right)+\left(b_{1}+\eta^{-}\right)(1-\theta) \tilde{a}}{b_{1} b_{2}+b_{2} \eta^{-}-\beta^{2}}
$$

as the optimal direct sale prices when $r=\bar{r}_{d}^{\mathrm{NI}}$ and $r=\underline{r}_{d}^{\mathrm{NI}}$, respectively.

If we can show $\hat{w}_{d}^{\mathrm{NI}}\left(\bar{r}_{d}^{\mathrm{NI}}\right)<\bar{r}_{d}^{\mathrm{NI}}, \hat{w}_{d}^{\mathrm{NI}}\left(\underline{r}_{d}^{\mathrm{NI}}\right)<\underline{r}_{d}^{\mathrm{NI}}, \hat{p}_{d, d}^{\mathrm{NI}}\left(\bar{r}_{d}^{\mathrm{NI}}\right)<\frac{2 \beta \bar{r}_{d}^{\mathrm{NI}}+(1-\theta) \tilde{a}}{2 b_{2}}$, and $\hat{p}_{d, d}^{\mathrm{NI}}\left(\underline{r}_{d}^{\mathrm{NI}}\right)<\frac{2 \beta \underline{r}_{d}^{\mathrm{NI}}+(1-\theta) \tilde{a}}{2 b_{2}}$. Then proof is completed. Because

$$
\hat{w}_{d}^{\mathrm{NI}}\left(\bar{r}_{d}^{\mathrm{NI}}\right)-\bar{r}_{d}^{\mathrm{NI}}=\frac{\theta a\left(\beta^{2}-b_{2}\left(b_{1}+\eta^{+}\right)\right)-\beta(1-\theta) \tilde{a} 0.5 \eta^{+}}{4\left(b_{1} b_{2}+b_{2} \eta^{+}-\beta^{2}\right) b_{1}+\left(b_{1} b_{2}+b_{2} \eta^{+}-3 \beta^{2}\right) \eta^{+}} \leq 0,
$$

where inequality follows from $b_{1}, b_{2}>\beta$. Similarly, we can prove $\hat{w}_{d}^{\mathrm{NI}}\left(\underline{r}_{d}^{\mathrm{NI}}\right)<\underline{r}_{d}^{\mathrm{NI}}$. Besides,

$$
\frac{2 \beta \bar{r}_{d}^{\mathrm{NI}}+(1-\theta) \tilde{a}}{2 b_{2}}-\hat{p}_{d, d}^{\mathrm{NI}}\left(\bar{r}_{d}^{\mathrm{NI}}\right)=\frac{2 \theta a \beta\left(b_{2}\left(b_{1}+\eta^{+}\right)-\beta^{2}\right)+\beta^{2}(1-\theta) \tilde{a} \eta^{+}}{2 b_{2}\left[4\left(b_{1} b_{2}+b_{2} \eta^{+}-\beta^{2}\right) b_{1}+\left(b_{1} b_{2}+b_{2} \eta^{+}-3 \beta^{2}\right) \eta^{+}\right]}>0 .
$$

Similarly, we obtain $\frac{2 \beta \underline{r}_{d}^{\mathrm{NI}}+(1-\theta) \tilde{a}}{2 b_{2}}-\hat{p}_{d, d}^{\mathrm{NI}}\left(\underline{r}_{d}^{\mathrm{NI}}\right)>0$. Proof is completed. 
Proof of Corollary 4.5. Because $\bar{p}_{r, c}^{\mathrm{NI}}$ and $\bar{p}_{d, c}^{\mathrm{NI}}$ are specific cases (i.e., $\left.\eta^{ \pm}=0\right)$ of $\hat{p}_{r, c}^{\mathrm{NI}}$ and $\hat{p}_{d, c}^{\mathrm{NI}}$, respectively, and $\hat{p}_{r, c}^{\mathrm{NI}}$ and $\hat{p}_{d, c}^{\mathrm{NI}}$ are nonincreasing in $\eta^{+}$and $\eta^{-}$(see Prop. 4.2 ), so do $\bar{p}_{r, c}^{\mathrm{NI}}$ and $\bar{p}_{d, c}^{\mathrm{NI}}$.

Proof of Proposition 4.6. It immediately follows from equation (4.3) that $\mathbb{E} \bar{\Pi}_{c}^{\mathrm{NI}}$ is independent of $r$, and $\mathbb{E} \tilde{\Pi}_{c}^{\mathrm{NI}}$ is linearly increasing in $r$. We prove the monotonicity of $\mathbb{E} \hat{\Pi}_{c}^{\mathrm{FI}}$ w.r.t. $r$. From equation (4.2), we have

$$
\mathbb{E} \hat{\Pi}_{c}^{\mathrm{NI}}=\hat{p}_{r, c}^{\mathrm{NI}}\left(\theta a-b_{1} \hat{p}_{r, c}^{\mathrm{NI}}+\beta \hat{p}_{d, c}^{\mathrm{NI}}\right)+\hat{p}_{r, c}^{\mathrm{NI}}\left((1-\theta) \tilde{a}-b_{2} \hat{p}_{d, c}^{\mathrm{NI}}+\beta \hat{p}_{r, c}^{\mathrm{NI}}\right) .
$$

Taking the first derivation of $\mathbb{E} \hat{\Pi}_{c}^{\mathrm{FI}}$ w.r.t. $r$, we obtain that if $r \geq \bar{r}_{c}^{\mathrm{NI}}$ or $r \leq \underline{r}_{c}^{\mathrm{NI}}$,

$$
\frac{\partial \mathbb{E} \hat{\Pi}_{c}^{\mathrm{NI}}}{\partial r}=\frac{\eta^{ \pm}\left(\theta a b_{2}+(1-\theta) \tilde{a} \beta+b_{2} \eta^{ \pm} r\right)}{2\left(b_{1}+\eta^{ \pm}\right) b_{2}-2 \beta^{2}}>0,
$$

where $\eta^{ \pm}$denotes $\eta^{+}$and $\eta^{-}$; if $\underline{r}_{c}^{\mathrm{NI}}<r<\bar{r}_{c}^{\mathrm{NI}}$,

$$
\frac{\partial \mathbb{E} \hat{\Pi}_{c}^{\mathrm{NI}}}{\partial r}=\frac{\theta a b_{2}+(1-\theta) \tilde{a} \beta}{b_{2}}-\frac{2 \beta^{2}-2 b_{1} b_{2}}{b_{2}} r>0,
$$

where the inequality follows from $r>\underline{r}_{c}^{\mathrm{NI}}=\frac{\theta a b_{2}+(1-\theta) a \beta}{2 b_{1} b_{2}+\eta^{-} b_{2}-2 \beta^{2}}$. Combining $\mathbb{E} \hat{\Pi}_{c}^{\mathrm{NI}}$ is continuous, we have $\mathbb{E} \hat{\Pi}_{c}^{\mathrm{NI}}$ is increasing in $r$. Clearly, when $r \leq \bar{p}_{r, c}^{\mathrm{NI}}, \tilde{D}_{r, c}^{\mathrm{NI}} \leq \bar{D}_{r, c}^{\mathrm{NI}}$ and when $r>\bar{p}_{r, c}^{\mathrm{NI}}, \tilde{D}_{r, c}^{\mathrm{NI}}>\bar{D}_{r, c}^{\mathrm{NI}}$. Hence, when $r \leq \bar{p}_{r, c}^{\mathrm{NI}}$,

$$
\mathbb{E} \bar{\Pi}_{c}^{\mathrm{NI}}=\bar{p}_{r, c}^{\mathrm{NI}} \bar{D}_{r, c}^{\mathrm{NI}}+\bar{p}_{d, c}^{\mathrm{NI}} \bar{D}_{d, c}^{\mathrm{NI}} \geq \mathbb{E} \tilde{\Pi}_{c}^{\mathrm{NI}}=\bar{p}_{r, c}^{\mathrm{NI}} \tilde{D}_{r, c}^{\mathrm{NI}}+\bar{p}_{d, c}^{\mathrm{NI}} \tilde{D}_{d, c}^{\mathrm{NI}}
$$

and when $r>\bar{p}_{r, c}^{\mathrm{NI}}, \mathbb{E}_{\bar{c}}^{\mathrm{NI}}<\mathbb{E} \tilde{\Pi}_{c}^{\mathrm{NI}}$.

Because $\bar{p}_{r, c}^{\mathrm{NI}}$ is the suboptimal solution of equation (4.2) and $\mathbb{E}_{\bar{\Pi}}^{\mathrm{NI}}$ is the corresponding suboptimal revenue, combining the uniqueness of the optimal solution (Prop. 4.1), we obtain $\mathbb{E} \hat{\Pi}_{c}^{\mathrm{NI}}$ is always greater than $\mathbb{E} \bar{\Pi}_{c}^{\mathrm{NI}}$.

Proof of Proposition 4.7. Readers can refer to the proof of Proposition 4.6.

Proof of Proposition 5.1. Similar to the proof of Proposition 4.1 except $\tilde{a}$ is replaced with $a$.

Proof of Proposition 5.2. Similar to the proof of Proposition 4.2 except $\tilde{a}$ is replaced with $a$, we could obviously obtain that $\hat{p}_{r, c}^{\mathrm{IS}}$ and $\hat{p}_{d, c}^{\mathrm{IS}}$, are (i) nonincreasing in $\eta^{+}$and $\eta^{-}$; (ii) increasing in $r$, the increment rate of $\hat{p}_{r, c}^{\mathrm{IS}}$ is larger than that of $\hat{p}_{d, c}^{\mathrm{IS}}$.

Proof of Proposition 5.3. Using backward induction. Given the manufacturer's decisions $p_{d, d}^{\mathrm{IS}}$ and $w_{d}^{\mathrm{IS}}$, the optimal retail price is given by computing the first-order derivative of $\Pi_{r, d}^{\mathrm{IS}}$ w.r.t. $p_{r, d}^{\mathrm{IS}}$, we obtain

$$
p_{r, d}^{\mathrm{IS}}=\frac{1}{2} \frac{\theta a+\eta r+k \beta+\beta p_{d, d}^{\mathrm{IS}}+\left(b_{1}+\eta\right)\left(w_{d}^{\mathrm{IS}}-k\right)}{b_{1}+\eta} .
$$

Substituting $p_{r, d}^{\mathrm{IS}}$ into $\Pi_{m}^{\mathrm{IS}}$ in equation (5.1), and take the partial derivatives of $\Pi_{m, d}^{\mathrm{IS}}$ w.r.t. $w_{d}^{\mathrm{IS}}$ and $p_{d, d}^{\mathrm{IS}}$, we have the following Hessian matrix

$$
\tilde{\mathrm{H}}^{\mathrm{IS}}=\left(\begin{array}{cc}
\frac{\partial^{2} \Pi_{m, d}^{\mathrm{IS}}}{\partial\left(w_{d}^{\mathrm{IS}}\right)^{2}} & \frac{\partial^{2} \Pi_{m, d}^{\mathrm{IS}}}{\partial\left(w_{d}^{\mathrm{IS}}\right) \partial\left(p_{d, d}^{\mathrm{IS}}\right)} \\
\frac{\partial^{2} \Pi_{m, d}^{\mathrm{IS}}}{\partial\left(p_{d, d}^{\mathrm{IS}}\right) \partial\left(w_{d}^{\mathrm{IS}}\right)} & \frac{\partial^{2} \Pi_{m, d}^{\mathrm{IS}}}{\partial\left(p_{d, d}^{\mathrm{IS}}\right)^{2}}
\end{array}\right)=\left(\begin{array}{cc}
-b_{1}-\eta & \beta \\
\beta & \frac{\beta^{2}}{b_{1}+\eta}-2 b_{2}
\end{array}\right) .
$$


Since $\frac{\partial^{2} \Pi_{m, d}^{\mathrm{IS}}}{\partial\left(w_{d}^{\mathrm{IS}}\right)^{2}}=-b_{1}-\eta<0$, and $\operatorname{det}\left(\tilde{\mathrm{H}}^{\mathrm{IS}}\right)=2\left(b_{1}+\eta\right) b_{2}-2 \beta^{2}>0$, and thus $\Pi_{m, d}^{\mathrm{IS}}$ is jointly concave in $w_{d}^{\mathrm{IS}}$ and $p_{d, d}^{\mathrm{IS}}$, i.e., $\Pi_{m, d}^{\mathrm{IS}}$ has a unique solution. Computing the first-order derivatives of $\Pi_{m, d}^{\mathrm{IS}}$ w.r.t. $w_{d}^{\mathrm{IS}}$ and $p_{d, d}^{\mathrm{IS}}$

$$
\begin{aligned}
\frac{\partial \Pi_{m, d}^{\mathrm{IS}}}{\partial w_{d}^{\mathrm{IS}}} & =\frac{1}{2}(\theta a+\eta r)+\beta\left(p_{d, d}^{\mathrm{IS}}-k\right)-\left(b_{1}+\eta\right)\left(w_{d}^{\mathrm{IS}}-k\right), \\
\frac{\partial \Pi_{m, d}^{\mathrm{IS}}}{\partial p_{d, d}^{\mathrm{IS}}} & =\beta\left(w_{d}^{\mathrm{IS}}-k\right)+\left(\frac{\beta^{2}}{b_{1}+\eta}-2 b_{2}\right) p_{d, d}^{\mathrm{IS}}+(1-\theta) a+\frac{\beta(\theta a+\eta r)}{2\left(b_{1}+\eta\right)}+b_{2} k .
\end{aligned}
$$

Setting the above formulae to zero, we obtain the optimal retail price and direct sale price

$$
\begin{aligned}
\hat{w}_{d}^{\mathrm{IS}} & =\frac{1}{2} \frac{(\theta a+\eta r) b_{2}+\left[(1-\theta) a-b_{2} k\right] \beta}{b_{1} b_{2}+b_{2} \eta-\beta^{2}}+\frac{1}{2} \frac{\beta^{3} k}{\left(b_{1} b_{2}+b_{2} \eta-\beta^{2}\right)\left(b_{1}+\eta\right)}+k, \\
\hat{p}_{d, d}^{\mathrm{IS}} & =\frac{1}{2} \frac{(\theta a+\eta r) \beta+\left[(1-\theta) a+b_{2} k\right]\left(b_{1}+\eta\right)-\beta^{2} k}{b_{1} b_{2}+b_{2} \eta-\beta^{2}} .
\end{aligned}
$$

Substituting $\hat{w}_{d}^{\mathrm{IS}}$ and $\hat{p}_{d, d}^{\mathrm{IS}}$ into equation (A.5), we get

$$
\hat{p}_{r, d}^{\mathrm{IS}}=\frac{(\theta a+\eta r)\left(3 b_{1} b_{2}+3 b_{2} \eta-\beta^{2}\right)+2(1-\theta) a \beta\left(b_{1}+\eta\right)}{4\left(b_{1} b_{2}+b_{2} \eta-\beta^{2}\right)\left(b_{1}+\eta\right)}+\frac{k \beta}{2\left(b_{1}+\eta\right)} .
$$

Similar to the analysis of the proof of Proposition 4.3, there still exists two thresholds on reference price $\bar{r}_{d}^{\mathrm{IS}}=\frac{\left.\theta a\left(3 b_{1} b_{2}+3 b_{2} \eta^{+}-\beta^{2}\right)+2(1-\theta) a \beta\left(b_{1}+\eta^{+}\right)+2 k \beta\left(b_{1} b_{2}+b_{2} \eta^{+}-\beta^{2}\right)\right)}{4\left(b_{1} b_{2}+b_{2} \eta^{+}-\beta^{2}\right) b_{1}+\left(b_{1} b_{2}+b_{2} \eta^{+}-3 \beta^{2}\right) \eta^{+}} \geq \underline{r}_{d}^{\text {IS }}=$ $\frac{\left.\theta a\left(3 b_{1} b_{2}+3 b_{2} \eta^{-}-\beta^{2}\right)+2(1-\theta) a \beta\left(b_{1}+\eta^{-}\right)+2 k \beta\left(b_{1} b_{2}+b_{2} \eta^{-}-\beta^{2}\right)\right)}{4\left(b_{1} b_{2}+b_{2} \eta^{-}-\beta^{2}\right) b_{1}+\left(b_{1} b_{2}+b_{2} \eta^{-}-3 \beta^{2}\right) \eta^{-}}$. When $r \geq \bar{r}_{d}^{\mathrm{IS}}$, the pricing decisions are independent of $\eta^{-}$; and when $r \leq \underline{r}_{d}^{\mathrm{IS}}$ the pricing decisions are independent of $\eta^{+}$; When $\underline{r}_{d}^{\mathrm{IS}} \leq r \leq \bar{r}_{d}^{\mathrm{IS}}$, the optimal retail price would be $r$. It follows from equation (A.5) that

$$
\hat{w}_{d}^{\mathrm{IS}}=k+2 r-\frac{\theta q+\eta r+k \beta+k \hat{p}_{d, d}^{\mathrm{IS}}}{b_{1}+\eta}
$$

then substituting $\hat{w}_{d}^{\mathrm{IS}}$ into equation (5.1) and maximizing $\Pi_{m}^{\mathrm{IS}}$, we obtain

$$
\begin{aligned}
\hat{w}_{d}^{\mathrm{IS}} & =\frac{2 b_{2}\left(b_{1} r-\theta a\right)-(1-\theta) a \beta-3 b_{2} k \beta}{2\left(\beta^{2}+b_{2}\left(b_{1}+\eta\right)\right)}-\frac{k \beta^{2}}{2\left(b_{1}+\eta\right)\left(\beta^{2}+b_{2}\left(b_{1}+\eta\right)\right)}+k, \\
\hat{p}_{d, d}^{\mathrm{IS}} & =\frac{2 \beta\left(\left(2 b_{1}+\eta\right) r-\theta a\right)+\left(b_{1}+\eta\right)\left((1-\theta) a+b_{2} k\right)-k \beta^{2}}{2\left(\beta^{2}+b_{2}\left(b_{1}+\eta\right)\right)} .
\end{aligned}
$$

Proof of Corollary 5.4. The proof is similar to the proof of Proposition 4.4. We omit the details.

Proof of Proposition 5.5. Computing the derivations of $\hat{w}_{d}^{\mathrm{IS}}, \hat{p}_{r, d}^{\mathrm{IS}}$ and $\hat{p}_{d, d}^{\mathrm{IS}}$ w.r.t. $k$, respectively. If $r \geq \bar{r}_{d}^{\mathrm{IS}}$,

$$
\frac{\partial \hat{w}_{d}^{\mathrm{IS}}}{\partial k}=1-\frac{\beta}{2\left(b_{1}+\eta^{+}\right)}>0, \frac{\partial \hat{p}_{d, d}^{\mathrm{IS}}}{\partial k}=\frac{1}{2}, \frac{\partial \hat{p}_{r, d}^{\mathrm{IS}}}{\partial k}=\frac{\beta}{2\left(b_{1}+\eta^{+}\right)}>0,
$$

where the inequalities follow from $b_{1}>\beta>0$. If $r \leq \underline{r}_{d}^{\mathrm{IS}}$,

$$
\frac{\partial \hat{w}_{d}^{\mathrm{IS}}}{\partial k}=\frac{1}{2} \frac{\left(b_{1} b_{2}+b_{2} \eta^{-}-\beta^{2}\right)\left(2 b_{1}+2 \eta^{-}-\beta\right)}{\left(b_{1} b_{2}+b_{2} \eta^{-}-\beta^{2}\right)\left(b_{1}+\eta^{-}\right)}>0, \frac{\partial \hat{p}_{d, d}^{\mathrm{IS}}}{\partial k}=\frac{1}{2}, \frac{\partial \hat{p}_{r, d}^{\mathrm{IS}}}{\partial k}=\frac{\beta}{2\left(b_{1}+\eta^{-}\right)}
$$


If $\underline{r}_{d}^{\mathrm{IS}}<r<\bar{r}_{d}^{\mathrm{IS}}$

$$
\frac{\partial \hat{w}_{d}^{\mathrm{IS}}}{\partial k}=\frac{\partial \hat{p}_{r, d}^{\mathrm{IS}}}{\partial k}=0, \frac{\partial \hat{p}_{d, d}^{\mathrm{IS}}}{\partial k}=\frac{b_{2}-\beta}{2 b_{2}}>0,
$$

where the inequality follows from $b_{2}>\beta$. Clearly, when $r \leq \underline{r}_{d}^{\mathrm{IS}}$ and $r \geq \bar{r}_{d}^{\mathrm{IS}}, \frac{\partial \hat{w}_{d}^{\mathrm{IS}}}{\partial k}>\frac{\partial \hat{p}_{d, d}^{\mathrm{IS}}}{\partial k}>\frac{\partial \hat{p}_{r, d}^{\mathrm{IS}}}{\partial k}$. Combining $\frac{\partial \hat{p}_{r, d}^{\mathrm{IS}}}{\partial k}, \frac{\partial \hat{w}_{d}^{\mathrm{IS}}}{\partial k}$ and $\frac{\partial \hat{p}_{r, d}^{\mathrm{S}}}{\partial k}$ are independent of $k$. We obtain $\frac{\partial \hat{p}_{d, d}^{\mathrm{IS}}}{\partial k}$ is strictly increasing in $k$.

Proof of Corollary 5.6. The proof is similar to the proof of Corollary 4.5. We omit the details.

Proof of Proposition 5.7. Similar to the proof of Proposition 4.6 except $\tilde{a}$ is replaced with $a$.

Proof of Proposition 5.8. We first show the manufacturer's optimal, expected and actual revenues, i.e., $\hat{\Pi}_{m, d}^{\mathrm{IS}}$, $\tilde{\Pi}_{m, d}^{\mathrm{IS}}$ and $\bar{\Pi}_{m, d}^{\mathrm{IS}}$, are decreasing in $k$. Then, the same method can be applied to obtain the monotonicity of the retailer's revenues $\hat{\Pi}_{r, d}^{\mathrm{IS}}, \tilde{\Pi}_{r, d}^{\mathrm{IS}}$ and $\bar{\Pi}_{r, d}^{\mathrm{IS}}$ w.r.t. $k . \bar{\Pi}_{m, d}^{\mathrm{IS}}$ decreases with increasing $k$ is immediately follows from equation (5.4) that $\bar{w}_{d}^{\mathrm{IS}}, \bar{D}_{r, d}^{\mathrm{IS}}, \bar{p}_{d, d}^{\mathrm{IS}}$ and $\bar{D}_{d, d}^{\mathrm{IS}}$ are independent of $k$. Taking the derivations of $\tilde{\Pi}_{m, d}^{\mathrm{IS}}$ and $\hat{\Pi}_{m, d}^{\mathrm{IS}}$ w.r.t. $k$, we have

$$
\frac{\partial \hat{\Pi}_{m, d}^{\mathrm{IS}}}{\partial k}=\left(\hat{p}_{d, d}-k\right) \frac{\beta^{2}-b_{2}\left(b_{1}+\eta\right)}{2\left(b_{1}+\eta\right)}-\frac{\beta}{2\left(b_{1}+\eta\right)} \hat{D}_{r, d}-\frac{1}{2} \hat{D}_{d, d}<0,
$$

where the inequality follows from $k<\hat{p}_{d, d}, \beta<b_{1}, b_{2}, \eta=\left\{\eta^{+}, \eta^{-}\right\}>0, \hat{D}_{r, d}=\theta a-b_{1} \hat{p}_{r, d}+\beta \hat{p}_{d, d}+\eta\left(r-\hat{p}_{r, d}\right)>$ and $\hat{D}_{d, d}=\theta a-b_{2} \hat{p}_{d, d}+\beta \hat{p}_{r, d}>0$; as well as

$$
\frac{\partial \tilde{\Pi}_{m, d}^{\mathrm{IS}}}{\partial k}=-\left(\tilde{D}_{r, d}+\tilde{D}_{r, d}\right)<0 .
$$

Note that the last inequality follows from the demands are nonnegative.

Proof of Corollary 5.9. The proof follows directly from Proposition 5.7. We omit the details.

\section{Appendix B.}

Here, we consider a case where the consumers respectively form two reference prices in the retail channel $r_{1}$ and direct channel $r_{2}$, then consumers' demand comes from two channels can be written as follows:

$$
\breve{D}_{r}=\theta a-b_{1} p_{r}+\beta p_{d}+\eta\left(r_{1}-p_{r}\right) \text { and } \breve{D}_{d}=(1-\theta) a-b_{2} p_{d}+\beta p_{r}+\eta\left(r_{2}-p_{d}\right) .
$$

Obviously, it will be extremely complicated if two separate reference prices are considered. Besides, consumers generally form the same reference price for two different channels in minds, which seems more realistic than form two separate reference prices, thus let $r_{1}=r_{2}=r$. Then, the individual and total revenues of the manufacturer and the retailer can be respectively determined as

$$
\breve{\Pi}_{m}=w \breve{D}_{r}+p_{d} \breve{D}_{d}, \breve{\Pi}_{r}=\left(p_{r}-w\right) \breve{D}_{r}, \breve{\Pi}=\breve{\Pi}_{m}+\breve{\Pi}_{r}=p_{r} \check{D}_{r}+p_{d} \breve{D}_{d}
$$

In the following, we take the centralized supply chain as an example to analyze the model. Computing the partial derivatives of $\breve{\Pi}_{c}$ w.r.t. $p_{r}$ and $p_{d}$, then we have the following Hessian matrix

$$
\breve{\mathrm{H}}=\left(\begin{array}{cc}
\frac{\partial^{2} \breve{\Pi}}{\partial\left(p_{r}\right)^{2}} & \frac{\partial^{2} \breve{\Pi}}{\partial\left(p_{r}\right) \partial\left(p_{d}\right)} \\
\frac{\partial^{2} \breve{\Pi}}{\partial\left(p_{r}\right) \partial\left(p_{d}\right)} & \frac{\partial^{2} \breve{\Pi}}{\partial\left(p_{d}\right)^{2}}
\end{array}\right)=\left(\begin{array}{cc}
-2 b_{1}-2 \eta & 2 \beta \\
2 \beta & -2 b_{2}-2 \eta
\end{array}\right) .
$$


Since $\frac{\partial^{2} \breve{\Pi}}{\partial\left(p_{r}\right)^{2}}=-2 b_{1}-2 \eta<0$ and $\operatorname{det}(\breve{H})=4\left(b_{1}+\eta\right)\left(b_{2}+\eta\right)-4 \beta^{2}>0$ (where the inequalities follow from $b_{1}, b_{2}>\beta>0$ and $\left.\eta \in\left\{\eta^{+}, \eta^{-}\right\}>0\right)$, thus $\breve{\Pi}$ is jointly concave in $p_{r}$ and $p_{d}$, which implies there exists a unique solution to maximize $\breve{\Pi}$. Using the first derivative condition, we obtain

$$
\begin{aligned}
& \breve{p}_{r}:=\breve{p}_{r}(\eta)=\frac{\theta a\left(b_{2}+\eta\right)+(1-\theta) a \beta+\eta r\left(b_{2}+\eta+\beta\right)}{2\left(b_{1}+\eta\right)\left(b_{2}+\eta\right)-2 \beta^{2}} \text { and } \\
& \breve{p}_{d}:=\breve{p}_{d}(\eta)=\frac{(1-\theta) a\left(b_{1}+\eta\right)+\beta \theta a+\eta r\left(b_{1}+\eta+\beta\right)}{2\left(b_{1}+\eta\right)\left(b_{2}+\eta\right)-2 \beta^{2}} .
\end{aligned}
$$

Let $\breve{p}_{r}=r$, we have $r(r ; \eta)=\frac{\theta a\left(b_{2}+\eta\right)+(1-\theta) a \beta}{2\left(b_{1}+\eta\right)\left(b_{2}+\eta\right)-2 \beta^{2}-\eta\left(b_{2}+\eta+\beta\right)}$. It could be proved that $r(\eta)$ is decreasing in $\eta$. Then, define

$$
\bar{r}_{r}=\frac{\theta a\left(b_{2}+\eta^{+}\right)+(1-\theta) a \beta}{2\left(b_{1}+\eta^{+}\right)\left(b_{2}+\eta^{+}\right)-2 \beta^{2}-\eta^{+}\left(b_{2}+\eta^{+}+\beta\right)}>\underline{r}_{r}=\frac{\theta a\left(b_{2}+\eta^{-}\right)+(1-\theta) a \beta}{2\left(b_{1}+\eta^{-}\right)\left(b_{2}+\eta^{-}\right)-2 \beta^{2}-\eta^{-}\left(b_{2}+\eta^{-}+\beta\right)},
$$

where the inequality follows from $\eta^{-}>\eta^{+}$. Similarly, let $\breve{p}_{d}=r$, we have $r(d ; \eta)=\frac{(1-\theta) a\left(b_{1}+\eta\right)+\beta \theta a}{2\left(b_{1}+\eta\right)\left(b_{2}+\eta\right)-2 \beta^{2}-\eta\left(b_{1}+\eta+\beta\right)}$ is decreasing in $\eta$. Then, define

$$
\bar{r}_{d}=\frac{(1-\theta) a\left(b_{1}+\eta^{+}\right)+\beta \theta a}{2\left(b_{1}+\eta^{+}\right)\left(b_{2}+\eta^{+}\right)-2 \beta^{2}-\eta^{+}\left(b_{1}+\eta^{+}+\beta\right)}>\underline{r}_{d}=\frac{(1-\theta) a\left(b_{1}+\eta^{-}\right)+\beta \theta a}{2\left(b_{1}+\eta^{-}\right)\left(b_{2}+\eta^{-}\right)-2 \beta^{2}-\eta^{-}\left(b_{1}+\eta^{-}+\beta\right)} .
$$

By comparing $\underline{r}_{r}, \underline{r}_{d}, \bar{r}_{r}, \bar{r}_{d}$, we discuss the optimal decisions in the following scenarios: (a) $\bar{r}_{d}<\underline{r}_{r}$; (b) $\underline{r}_{d}<\underline{r}_{r}<\bar{r}_{d}<\bar{r}_{r} ;$ (c) $\underline{r}_{d}<\underline{r}_{r}<\bar{r}_{r}<\bar{r}_{d}$; (d) $\underline{r}_{r}<\underline{r}_{d}<\bar{r}_{d}<\bar{r}_{r} ;$ (e) $\underline{r}_{r}<\underline{r}_{d}<\bar{r}_{r}<\bar{r}_{d}$; (f) $\bar{r}_{r}<\underline{r}_{d}$.

In scenario (a), from the model setting, we know that if the observed reference price is larger than the selling price, then $\eta=\eta^{+}$, otherwise $\eta=\eta^{-}$, which implies that (i) if $r>\bar{r}_{r}, p_{r}^{*}=\breve{p}_{r}\left(\eta^{+}\right)$and $p_{d}^{*}=\breve{p}_{d}\left(\eta^{+}\right)$, where $p_{r}^{*}$ and $p_{d}^{*}$ denotes the optimal retail price and the optimal direct price; (ii) If $r<\underline{r}_{d}, p_{r}^{*}=\breve{p}_{r}\left(\eta^{-}\right)$and $p_{d}^{*}=\breve{p}_{d}\left(\eta^{-}\right)$; (iii) $\underline{r}_{r}<r \leq \bar{r}_{r}, p_{r}^{*}=r$ and $p_{d}^{*}=\breve{p}_{d}\left(\eta^{+}\right)$; (iv) If $\bar{r}_{d}<r \leq \underline{r}_{r}, p_{r}^{*}=\breve{p}_{r}\left(\eta^{-}\right)$and $p_{d}^{*}=\breve{p}_{d}\left(\eta^{+}\right)$; (v) $\underline{r}_{d} \leq r \leq \bar{r}_{d}$, $p_{r}^{*}=\breve{p}_{r}\left(\eta^{-}\right)$and $p_{d}^{*}=r$.

In scenario (b), using the same method in scenario (a), we obtain that (i) if $r>\bar{r}_{r}, p_{r}^{*}=\breve{p}_{r}\left(\eta^{+}\right)$and $p_{d}^{*}=\breve{p}_{d}\left(\eta^{+}\right)$; (ii) If $r<\underline{r}_{d}, p_{r}^{*}=\breve{p}_{r}\left(\eta^{-}\right)$and $p_{d}^{*}=\breve{p}_{d}\left(\eta^{-}\right)$; (iii) $\bar{r}_{d}<r \leq \bar{r}_{r}, p_{r}^{*}=r$ and $p_{d}^{*}=\breve{p}_{d}\left(\eta^{+}\right.$); (iv) If $\underline{r}_{r}<r \leq \bar{r}_{d}, p_{r}^{*}=r$ and $p_{d}^{*}=r ;(\mathrm{v}) \underline{r}_{d} \leq r \leq \underline{r}_{r}, p_{r}^{*}=\breve{p}_{r}\left(\eta^{-}\right)$and $p_{d}^{*}=r$.

In scenario (c), the optimal pricing decisions are (i) if $r>\bar{r}_{d}, p_{r}^{*}=\breve{p}_{r}\left(\eta^{+}\right)$and $p_{d}^{*}=\breve{p}_{d}\left(\eta^{+}\right)$; (ii) If $r<\underline{r}_{d}$, $p_{r}^{*}=\breve{p}_{r}\left(\eta^{-}\right)$and $p_{d}^{*}=\breve{p}_{d}\left(\eta^{-}\right)$; (iii) $\bar{r}_{r}<r \leq \bar{r}_{d}, p_{r}^{*}=\breve{p}_{r}\left(\eta^{+}\right)$and $p_{d}^{*}=r$; (iv) If $\underline{r}_{r}<r \leq \bar{r}_{r}, p_{r}^{*}=r$ and $p_{d}^{*}=r$; (v) $\underline{r}_{d} \leq r \leq \underline{r}_{r}, p_{r}^{*}=\breve{p}_{r}\left(\eta^{-}\right)$and $p_{d}^{*}=r$.

In scenario (d), the optimal pricing decisions are (i) if $r>\bar{r}_{r}, p_{r}^{*}=\breve{p}_{r}\left(\eta^{+}\right)$and $p_{d}^{*}=\breve{p}_{d}\left(\eta^{+}\right.$); (ii) If $r<\underline{r}_{r}$, $p_{r}^{*}=\breve{p}_{r}\left(\eta^{-}\right)$and $p_{d}^{*}=\breve{p}_{d}\left(\eta^{-}\right)$; (iii) $\bar{r}_{d}<r \leq \bar{r}_{r}, p_{r}^{*}=r$ and $p_{d}^{*}=\breve{p}_{d}\left(\eta^{+}\right)$; (iv) If $\underline{r}_{d}<r \leq \bar{r}_{d}, p_{r}^{*}=r$ and $p_{d}^{*}=r$; (v) $\underline{r}_{r} \leq r \leq \underline{r}_{d}, p_{r}^{*}=r$ and $p_{d}^{*}=\breve{p}_{d}\left(\eta^{-}\right)$.

In scenario (e), the optimal pricing decisions are (i) if $r>\bar{r}_{d}, p_{r}^{*}=\breve{p}_{r}\left(\eta^{+}\right)$and $p_{d}^{*}=\breve{p}_{d}\left(\eta^{+}\right)$; (ii) If $r<\underline{r}_{r}$, $p_{r}^{*}=\breve{p}_{r}\left(\eta^{-}\right)$and $p_{d}^{*}=\breve{p}_{d}\left(\eta^{-}\right)$; (iii) $\bar{r}_{r}<r \leq \bar{r}_{d}, p_{r}^{*}=\breve{p}_{r}\left(\eta^{+}\right)$and $p_{d}^{*}=r$; (iv) If $\underline{r}_{d}<r \leq \bar{r}_{r}, p_{r}^{*}=r$ and $p_{d}^{*}=r$; (v) $\underline{r}_{r} \leq r \leq \underline{r}_{d}, p_{r}^{*}=r$ and $p_{d}^{*}=\breve{p}_{d}\left(\eta^{-}\right)$.

In scenario (f), the optimal pricing decisions are (i) if $r>\bar{r}_{d}, p_{r}^{*}=\breve{p}_{r}\left(\eta^{+}\right)$and $p_{d}^{*}=\breve{p}_{d}\left(\eta^{+}\right)$; (ii) If $r<\underline{r}_{r}$, $p_{r}^{*}=\breve{p}_{r}\left(\eta^{-}\right)$and $p_{d}^{*}=\breve{p}_{d}\left(\eta^{-}\right)$; (iii) $\underline{r}_{d}<r \leq \bar{r}_{d}, p_{r}^{*}=\breve{p}_{r}\left(\eta^{+}\right)$and $p_{d}^{*}=r$; (iv) If $\bar{r}_{r}<r \leq \underline{r}_{d}, p_{r}^{*}=\breve{p}_{r}\left(\eta^{+}\right)$and $p_{d}^{*}=\breve{p}_{d}\left(\eta^{-}\right) ;(\mathrm{v}) \underline{r}_{r} \leq r \leq \bar{r}_{r}, p_{r}^{*}=r$ and $p_{d}^{*}=\breve{p}_{d}\left(\eta^{-}\right)$.

Comparing equation (B.1) with equation (A.1), we find that the structures of the optimal prices are similar, except that some parameters need to be modified suitably, which implies that considering a reference price in direct channel has little influence on the exploration of ARE. However, in view of the above intricate optimal pricing decision (two thresholds on reference price will be formed when judging the optimal direct selling price), considering reference effect in the direct channel will complicate the analysis substantially, especially when analyzing the optimal decisions in the decentralized scenario and exploring the value of information sharing. The optimal solutions will be more complex when examining the value of ARE in section "Model misspecification". 
For a better understanding the influences of ARE on the pricing decisions and revenues (which is the main research objective in this paper), we do not consider the reference price in the direct channel.

Acknowledgements. This work was supported by National Natural Science Foundation of China (nos. 72001064, 72101242, 72171219, and 71971203), the China Postdoctoral Science Foundation (no. 2020M682053), and the Fundamental Research Funds for the Central Universities (nos. JS2020HGXJ0006, JZ2020HGQA0170, and WK2040000041).

\section{REFERENCES}

[1] G.P. Cachon and M. Fisher, Supply chain inventory management and the value of shared information. Manage. Sci. 46 (2000) $1032-1048$.

[2] P. Cao, N. Zhao and J. Wu, Dynamic pricing with bayesian demand learning and reference price effect. Eur. J. Oper. Res. 279 (2019) 540-556.

[3] X. Chen, P. Hu, S. Shum and Y. Zhang, Dynamic stochastic inventory management with reference price effects. Oper. Res. 64 (2016) 1529-1536.

[4] J. Chen, L. Liang, D.Q. Yao and S. Sun, Price and quality decisions in dual-channel supply chains. Eur. J. Oper. Res. 259 (2017) 935-948.

[5] J. Chen, W. Zhang and Z. Liu, Joint pricing, services and quality decisions in a dual-channel supply chain. RAIRO-Oper. Res. 54 (2020) 1041-1056.

[6] W.Y.K. Chiang, D. Chhajed and J.D. Hess, Direct marketing, indirect profits: a strategic analysis of dual-channel supply-chain design. Manage. Sci. 49 (2003) 1-20.

[7] Y. Duan and Y. Cao, Optimal pricing and deteriorating inventory control when inventory stimulates stochastic demand with reference price effect. RAIRO-Oper. Res. 55 (2021) S1803-S1821.

[8] C.Y. Dye and C.T. Yang, Optimal dynamic pricing and preservation technology investment for deteriorating products with reference price effects. Omega 62 (2016) 52-67.

[9] G. Fibich, A. Gavious and O. Lowengart, Explicit solutions of optimization models and differential games with nonsmooth (asymmetric) reference-price effects. Oper. Res. 51 (2003) 721-734.

[10] M.G. Güler, T. Bilgiç and R. Güllü, Joint pricing and inventory control for additive demand models with reference effects. Ann. Oper. Res. 226 (2015) 255-276.

[11] D. Hanover, It's not a threat, just a promise. Chain Store Age 75 (1999) 176-176.

[12] T. Hosoda, M.M. Naim, S.M. Disney and A. Potter, Is there a benefit to sharing market sales information? linking theory and practice. Comput. Ind. Eng. 54 (2008) 315-326.

[13] G. Hua, S. Wang and T.E. Cheng, Price and lead time decisions in dual-channel supply chains. Eur. J. Oper. Res. 205 (2010) $113-126$.

[14] W. Huang and J.M. Swaminathan, Introduction of a second channel: implications for pricing and profits. Eur. J. Oper. Res. 194 (2009) 258-279.

[15] S. Huang, X. Guan and Y. Chen, Retailer information sharing with supplier encroachment. Prod. Oper. Manage. 27 (2018) 1133-1147.

[16] M.U. Kalwani, C.K. Yim, H.J. Rinne and Y. Sugita, A price expectations model of customer brand choice. J. Marketing Res. 27 (1990) 251-262.

[17] P.K. Kopalle, A.G. Rao and J.L. Assuncao, Asymmetric reference price effects and dynamic pricing policies. Marketing Sci. 15 (1996) 60-85.

[18] H.L. Lee, K.C. So and C.S. Tang, The value of information sharing in a two-level supply chain. Manage. Sci. 46 (2000) 626-643.

[19] M. Leng and M. Parlar, Allocation of cost savings in a three-level supply chain with demand information sharing: a cooperativegame approach. Oper. Res. 57 (2009) 200-213.

[20] B. Li, P. Chen, Q. Li and W. Wang, Dual-channel supply chain pricing decisions with a risk-averse retailer. Int. J. Prod. Res. $52(2014) 7132-7147$.

[21] L. Ma, Y. Zhao, W. Xue, T. Cheng and H. Yan, Loss-averse newsvendor model with two ordering opportunities and market information updating. Int. J. Prod. Econ. 140 (2012) 912-921.

[22] T. Mazumdar, S.P. Raj and I. Sinha, Reference price research: review and propositions. J. Marketing 69 (2005) 84-102.

[23] M. Rached, Z. Bahroun and J. Campagne, Assessing the value of information sharing and its impact on the performance of the various partners in supply chains. Comput. Ind. Eng. 88 (2015) 237-253.

[24] J.S. Raju and A. Roy, Market information and firm performance. Manage. Sci. 46 (2000) 1075-1084.

[25] X. Shen, L. Bao and Y. Yu, Coordinating inventory and pricing decisions with general price-dependent demands. Prod. Oper. Manage. 27 (2018) 1355-1367.

[26] C.S. Signorino and K. Yilmaz, Strategic misspecification in regression models. Am. J. Political Sci. 47 (2003) 551-566.

[27] L. Sun, X. Jiao, X. Guo and Y. Yu, Pricing policies in dual distribution channels: the reference effect of official prices. Eur. J. Oper. Res. 296 (2022) 146-157.

[28] A. Tversky and D. Kahneman, Prospect theory: an analysis of decision under risk. Econometrica 47 (1979) $263-291$. 
[29] J.-J. Wang, J. Dong, X. Yue and Q. Zhong, Information sharing in a supply chain with a coopetitive contract manufacturer. IEEE Trans. Syst. Man Cybern. Syst. 99 (2018) 1-17.

[30] N. Wang, T. Zhang, X. Zhu and P. Li, Online-offline competitive pricing with reference price effect. J. Oper. Res. Soc. 72 (2021) 642-653.

[31] M. Xue, W. Tang and J. Zhang, Optimal dynamic pricing for deteriorating items with reference-price effects. Int. J. Syst. Sci. 47 (2016) 2022-2031.

[32] X. Yue and J. Liu, Demand forecast sharing in a dual-channel supply chain. Eur. J. Oper. Res. 174 (2006) 646-667.

[33] J. Zhang, Q. Gou, L. Liang and Z. Huang, Supply chain coordination through cooperative advertising with reference price effect. Omega 41 (2013) 345-353.

[34] Q. Zhang, J. Zhang and W. Tang, A dynamic advertising model with reference price effect. RAIRO-Oper. Res. 49 (2015) 669-688.

[35] P. Zhang, Y. Xiong, Z. Xiong and Y. Zhou, Information sharing and service channel design in the presence of forecasting demand. J. Oper. Res. Soc. 69 (2018) 1920-1934.

[36] N. Zhao, Q. Wang, P. Cao and J. Wu, Dynamic pricing with reference price effect and price-matching policy in the presence of strategic consumers. J. Oper. Res. Soc. 70 (2019) 2069-2083.

[37] N. Zhao, Q. Wang and J. Wu, Optimal pricing and ordering decisions with reference effect and quick replenishment policy. Int. Trans. Oper. Res. 29 (2022) 1188-1219.

[38] X. Zhu, Outsourcing management under various demand information sharing scenarios. Ann. Oper. Res. 257 (2017) $449-467$.

\section{Subscribe to Open (S2O) A fair and sustainable open access model}

This journal is currently published in open access under a Subscribe-to-Open model (S2O). S2O is a transformative model that aims to move subscription journals to open access. Open access is the free, immediate, online availability of research articles combined with the rights to use these articles fully in the digital environment. We are thankful to our subscribers and sponsors for making it possible to publish this journal in open access, free of charge for authors.

\section{Please help to maintain this journal in open access!}

Check that your library subscribes to the journal, or make a personal donation to the S2O programme, by contacting subscribers@edpsciences.org

More information, including a list of sponsors and a financial transparency report, available at: https://www. edpsciences.org/en/maths-s2o-programme 